\title{
Light elements synthesized in the He-layer and the H-rich envelope of a type II supernova, II-Influence of initial chemical compositions-
}

\author{
Takashi Yoshida*, Kiyoshi Nakazawa, and Hiroyuki Emori \\ Department of Earth and Planetary Sciences, Tokyo Institute of Technology, \\ Meguro-ku, Tokyo 152-8551, Japan
}

(Received October 14, 1999; Revised March 1, 2000; Accepted March 8, 2000)

\begin{abstract}
This is the second paper of a series of our studies, in which we have investigated the light element synthesis (Li, $\mathrm{Be}, \mathrm{B}$, and the $\mathrm{CNO}$-elements) in the He-layer and the $\mathrm{H}$-rich envelope of supernovae and the aim of this paper is to see the sensitivity of the element synthesis to the chemical compositions in the presupernova stage. First, we deduced the probable range of the chemical composition in the presupernova stage on the basis of the previous studies of stellar evolution as well as the observational studies of stellar chemical compositions. Secondly, we pursued the explosive nucleosynthesis by using artificially modeled chemical compositions composed of only four elements $\left({ }^{1} \mathrm{H}\right.$, ${ }^{4} \mathrm{He},{ }^{12} \mathrm{C}$, and ${ }^{16} \mathrm{O}$ ). Combining these results, we constructed five kinds of diagrams between two isotopic/elemental ratios which are useful for distinguishing presolar grains of supernova origin: ${ }^{6} \mathrm{Li} /{ }^{16} \mathrm{O}$ and ${ }^{9} \mathrm{Be} /{ }^{16} \mathrm{O}$ to ${ }^{7} \mathrm{Li} /{ }^{16} \mathrm{O}$, and ${ }^{11} \mathrm{~B} /{ }^{7} \mathrm{Li},{ }^{14} \mathrm{~N} /{ }^{15} \mathrm{~N}$, and ${ }^{16} \mathrm{O} /{ }^{17} \mathrm{O}$ to ${ }^{12} \mathrm{C} /{ }^{13} \mathrm{C}$. In conclusion, the uncertainties in the chemical compositions in the presupernova stage brought smaller varieties in the isotopic/elemental ratios of the light elements compared with the varieties investigated in our first paper about the neutrino emission models.
\end{abstract}

\section{Introduction}

In the last decade we have identified a lot of presolar grains in which we observe several orders of magnitude of isotopic heterogeneity and, nowadays, it is believed that traces of their birth remain in these grains (e.g., Zinner, 1998). Until now several candidates are proposed as their origin, such as supernovae and AGB stars. Most studies on identifying presolar grains originated from supernovae have been conducted in Washington Univ. group (e.g., Zinner, 1998). Some presolar grains have been identified as supernova origin on the basis of the wide range of the carbon isotopic ratios, the ${ }^{28} \mathrm{Si}$ excesses, and the ${ }^{44}$ Ca excesses (e.g., Amari et al., 1992; Nittler et al., 1996). However, the number of studies on the quantitative classification of the presolar grains is small. Mainly Travaglio et al. (1999) quantitatively compared the isotopic ratios of the elements in the supernova originated presolar grains with those predicted from theoretical study of supernova nucleosynthesis but they did not definite regions of the isotopic ratios of the elements produced in the supernovae. On the other hand, nucleosynthesis in supernova explosions has been intensively studied by Woosley and his collaborators (e.g., Weaver and Woosley, 1980; Woosley and Weaver, 1986; Woosley et al., 1990; Woosley and Weaver, 1995; Meyer et al., 1995) and Nomoto and his collaborators (e.g., Nomoto et al., 1984; Shigeyama et al., 1987; Thielemann et al., 1990, 1996; Iwamoto et al., 1997). However, their

\footnotetext{
*Now at Department of Physics, Kyushu University, Chuo-ku, Fukuoka 810-8560, Japan

Copy right (C) The Society of Geomagnetism and Earth, Planetary and Space Sciences (SGEPSS); The Seismological Society of Japan; The Volcanological Society of Japan; The Geodetic Society of Japan; The Japanese Society for Planetary Sciences.
}

main interests are in the explanation of observational features of special supernova events or in construction of a new scenario of galactic chemical evolution. So, we need more precise study of the supernova nucleosynthesis aiming at an establishment of the theoretical basis to distinguish presolar grains of supernova origin.

In a series of our papers we have investigated the explosive nucleosynthesis of light elements (i.e., the $X$-elements and the CNO-elements) under various physical/chemical conditions and have found common features of the abundances as well as the elemental ratios of the light elements synthesized in the supernova explosion. In the last paper (Yoshida et al., 2000, which hereafter is referred to Paper I), we investigated the light element nucleosynthesis in the He-layer and the H-rich envelope of a $16.2 M_{\odot}$ supernova, taking account of a variety of the neutrino emission models (Woosley et al., 1990), i.e., the decay time of the neutrino flux, $\tau_{\nu}$, and the total energy carried by neutrinos, $E_{v}$. The main conclusions of Paper I are described as follows:

1) The amounts of ${ }^{6} \mathrm{Li}$ and ${ }^{9} \mathrm{Be}$ produced in the exploding He-layer and the H-rich envelope are much smaller than that of, for example, ${ }^{7} \mathrm{Li}$. The number ratios of ${ }^{6} \mathrm{Li} /{ }^{7} \mathrm{Li}$ and ${ }^{9} \mathrm{Be} /{ }^{7} \mathrm{Li}$ in the supernova ejecta are much smaller than the corresponding solar-system values.

2) Among the $X$-elements, ${ }^{7} \mathrm{Li},{ }^{10} \mathrm{~B}$, and ${ }^{11} \mathrm{~B}$ are synthesized appreciably in the He-layer and the inner H-rich envelope of a supernova $\left({ }^{10} \mathrm{~B}\right.$ is decomposed in the inner H-rich envelope). Although the amounts of these elements depend on $\tau_{v}$ and $E_{v}$, the ratio of ${ }^{11} \mathrm{~B} /{ }^{7} \mathrm{Li}$ is confined within a relatively small range. 
3) The abundances of ${ }^{12} \mathrm{C}$ and ${ }^{16} \mathrm{O}$ are completely determined by their initial values. Except in the innermost region of the H-rich envelope, so does the abundance of ${ }^{18} \mathrm{O}$.

4) The other CNO-elements, ${ }^{13} \mathrm{C},{ }^{14} \mathrm{~N},{ }^{15} \mathrm{~N}$, and ${ }^{17} \mathrm{O}$ are synthesized in the convective He-layer (in the other regions they keep their initial abundances). The amounts of these elements depend on the adopted neutrino emission models (i.e., $\tau_{v}$ and $E_{v}$ ) but are confined within a factor of 10 or so.

5) Presolar grains originated from supernovae are certainly distinguishable by the use of combination of the ratios of ${ }^{6} \mathrm{Li} /{ }^{7} \mathrm{Li},{ }^{9} \mathrm{Be} /{ }^{7} \mathrm{Li},{ }^{12} \mathrm{C} /{ }^{13} \mathrm{C},{ }^{14} \mathrm{~N} /{ }^{15} \mathrm{~N}$, and ${ }^{16} \mathrm{O} /{ }^{17} \mathrm{O}$.

In Paper I, we focused to the sensitivity of the element production to the adopted models of the neutrino emission. As pointed out in it, the above conclusions may be modified by the other physical/chemical conditions, such as the initial chemical compositions or mixing between distinct layers. So, in this paper we will investigate how the light element production responses to the adopted chemical compositions in the presupernova stage and modify a part of the conclusions found in Paper I if necessary.

In Section 2, the chemical composition in the presupernova stage will be presumed on the basis of studies on the stellar evolution and atmospheric chemical compositions of stars. In Section 3, we will describe in short an explosion model of a $16.2 M_{\odot}$ star and a nuclear reaction network constructed in Paper I. Furthermore, we will explain adopted models of the initial chemical compositions in the presupernova stage. In the present study, we will set up the models of the initial chemical compositions in the He-layer and the $\mathrm{H}$-rich envelope that consist of only four elements, ${ }^{1} \mathrm{H},{ }^{4} \mathrm{He}$, ${ }^{12} \mathrm{C}$, and ${ }^{16} \mathrm{O}$ with various contents. In addition, in order to see the degree of decomposition of pre-existing elements, we will also consider an artificial case where there are appreciable amounts of the $X$-elements and the rare CNO-elements $\left({ }^{13} \mathrm{C},{ }^{14} \mathrm{~N},{ }^{15} \mathrm{~N},{ }^{17} \mathrm{O}\right.$, and $\left.{ }^{18} \mathrm{O}\right)$ in the presupernova stage. In Section 4, results of numerical simulations will be shown: we will describe in detail the sensitivity of the light element production to the adopted initial chemical compositions. In Section 5, comparing the initial amounts of the light elements expected in the presupernova stage with those produced during the explosion, we will evaluate probable ranges of the isotopic/elemental ratios of the light elements observed in supernova ejecta. In Section 6, we will summarize briefly the results obtained in Sections 2, 4, and 5 and confirm the conclusions of Paper I on chief points.

\section{Probable Chemical Composition in the Presu- pernova Stage}

The light elements, which are produced on the way of the stellar evolution to the presupernova stage, affect, more or less, the nucleosynthesis during the supernova explosion by two ways; one is that relatively abundant elements govern the production and/or decomposition processes and the other is that the pre-existing light elements survive after the supernova explosion. So, it is important to evaluate probable values of the amounts and the isotopic ratios of the light
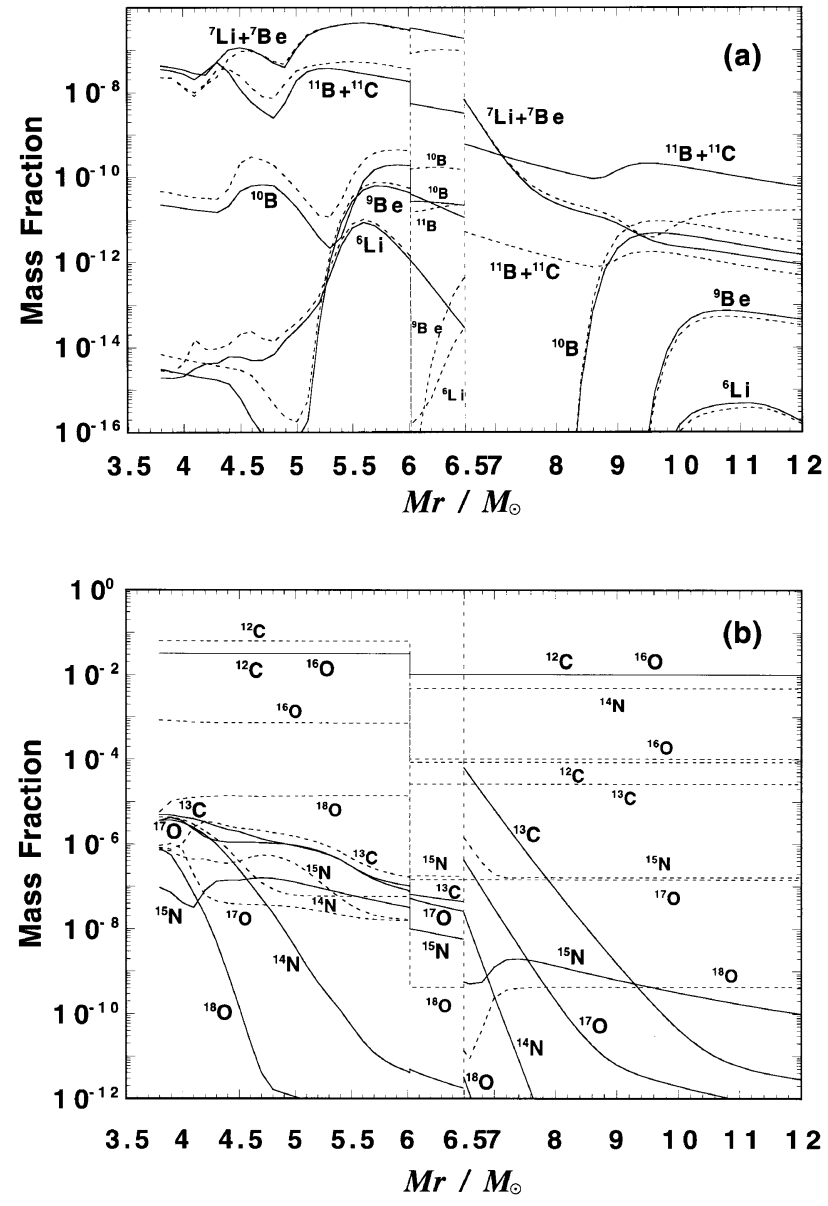

Fig. 1. Distributions of the final abundances of the $X$-elements (panel(a)) and the CNO-elements (panel(b)). Horizontal axis, $M_{r}$, denotes the internal mass coordinate (see Paper I). Solid lines and dashed lines show the mass fractions in model SII and model SI, respectively (see also Table 5). Discontinuities of the lines appear at the boundaries between the convective and the radiative He-layers $\left(M_{r}=6.0 M_{\odot}\right)$ and between the radiative He-layer and the H-rich envelope $\left(M_{r}=6.5 M_{\odot}\right)$.

elements in the He-layer and the H-rich envelope in order to see the reliable light element composition of the supernova ejecta.

\subsection{The CNO-elements}

First, we consider the abundances of the CNO-elements in the H-rich envelope. According to Lamb et al. (1976), during the time when a $25 M_{\odot}$ star evolves from the helium burning stage to the carbon burning stage, the temperature of the H-burning shell varies from $3.0 \times 10^{7} \mathrm{~K}$ to $5.0 \times 10^{7}$ $\mathrm{K}$. Using this temperature range and assuming that the corresponding density is in the range between $0.1 \mathrm{~g} / \mathrm{cm}^{3}$ and $10 \mathrm{~g} / \mathrm{cm}^{3}$ and the metallicity is $2 \%$ by weight, we evaluate possible ranges of the steady-flow equilibrium abundances of the CNO-elements. In this calculation we used a small nuclear reaction network in Fig. 1 of Paper I. The possible ranges of the elemental abundances of the major components and the isotopic ratios are tabulated in Table 1 as "equilibrium values" (the He content is assumed to be 0.25 to 0.60 ). One of the most remarkable points is that ${ }^{14} \mathrm{~N}$ is the most abundant element among the CNO-elements. For comparison, numerical results found by Woosley et al. (1990) and Weaver and Woosley (1993) (which hereafter are referred to 
W90 and WW93, respectively) are also shown in Table 1. The values due to WW93 are those in the presupernova stage of a $25 M_{\odot}$ star found from their evolutionary calculation. In WW93, the contents of some isotopes are beyond the ranges of the steady-flow equilibrium abundances probably because the CNO-cycle may not be in the steady-flow equilibrium. On the other hand, W90 gives the abundances consistent with those predicted by the steady-flow equilibrium condition. For the later use, we will define probable ranges of the contents of the CNO-elements in the H-rich envelope of a progenitor; probable ranges are determined so as to include all the values of the three examples (except ${ }^{17} \mathrm{O}$; see the next paragraph) and are shown in Table 1 as the "probable values".

It should be noted that the amount of ${ }^{17} \mathrm{O}$ given by WW93 is rather large compared with that of the steady-flow equilibrium value in this study. Almost all of ${ }^{17} \mathrm{O}$ is produced through ${ }^{16} \mathrm{O}(p, \gamma){ }^{17} \mathrm{~F}\left(, e^{+} v_{\mathrm{e}}\right){ }^{17} \mathrm{O}$ and decomposed by ${ }^{17} \mathrm{O}(p$, $\alpha)^{14} \mathrm{~N}$ at the H-burning shell (Woosley and Weaver, 1995). The reaction rate of ${ }^{17} \mathrm{O}(p, \alpha){ }^{14} \mathrm{~N}$ used in WW93 (Caughlan and Fowler, 1988) is much smaller than that of Landré $e$ t al. (1990) which we adopted. Woosley et al. (1997) pointed out that the total amount of ${ }^{17} \mathrm{O}$ of a $25 M_{\odot}$ supernova ejecta (Model S25A in Woosley and Weaver, 1995) decreases by a factor of 14 by the use of the reaction rate of Landré et al. (1990) and Blackmon et al. (1995) compared with that by the use of Caughlan and Fowler (1988). So, the amount of ${ }^{17} \mathrm{O}$ given by WW93 is reduced by a factor of 14 in evaluating the probable values in Table 1 (as well as in Tables 2 and 3).

In the radiative He-layer, it is natural to consider that the initial abundances are those of ashes of the hydrogen burning. In Table 2, the steady-flow equilibrium abundances of the CNO-cycle, calculated by the same way as earlier (but the He-content is assumed to be 0.95 to 0.97 ), are shown together with those of W90 and WW93. The probable abundances in the radiative He-layer are deduced from the three examples.

In the convective He-layer, the probable amounts of the CNO-elements (except ${ }^{14} \mathrm{~N}$ ) are deduced from W90, WW93, and Paper I by the similar way as earlier. They are tabulated in Table 3. Note that the three references give quite different values in the abundance of ${ }^{14} \mathrm{~N}$ from each other. Especially, the amount of ${ }^{14} \mathrm{~N}$ given by WW93 is extraordinarily large. In the typical temperature range of the He-burning $\left(\sim 2 \times 10^{8}\right.$ K) ${ }^{14} \mathrm{~N}$ reacts with ${ }^{4} \mathrm{He}$ very rapidly to form ${ }^{18} \mathrm{~F}$ compared with the triple $\alpha$-reaction. So, it is reasonable to expect that in the presupernova stage only a small amount of ${ }^{14} \mathrm{~N}$ is left unless there happens to occur, for example, a large scale mixing through which ${ }^{14} \mathrm{~N}$ is newly formed just before the supernova explosion. In fact, in $6 M_{\odot}$ and $8 M_{\odot} \mathrm{He}-$ star models which correspond to $20 M_{\odot}$ and $25 M_{\odot}$ main sequence stars, respectively (Nomoto and Hashimoto, 1988), very small amount of ${ }^{14} \mathrm{~N}$ (compared with that of WW93) is left in the presupernova stage (Hashimoto, 1999). Thus, the initial amount of ${ }^{14} \mathrm{~N}$ is deduced only from W90 and Paper I. The probable mass fraction of ${ }^{18} \mathrm{O}$ is adopted from the result of Paper I neglecting those of W90 and WW93 which are much larger than ours. This is because the reaction rate of ${ }^{18} \mathrm{O}(\alpha, \gamma){ }^{22} \mathrm{Ne}$ increases recently by a factor of 10 to 200 in the temperature range of the He-burning (Giesen et al., 1994) compared with that used in W90 and WW93.

\subsection{The $X$-elements}

It is reasonable to presume that, in the presupernova stage, there are no $X$-elements in the He-layer as well as in the inner (convective) H-rich envelope because they are all decomposed at the H-burning shell. Even if they existed in these regions, they would be eliminated by the nuclear processes during the supernova explosion as we will show in Section 4.3. Thus, we are interested in the initial abundances of the $X$-elements only in the outer H-rich envelope.

The initial abundances of the $X$-elements can be deduced only on the basis of observations of the atmospheric chemical compositions of stars. For Li, unfortunately, we have no definite answer although many observations have been made for various kinds of stellar objects (e.g., Andersen et al., 1984; Rebolo et al., 1986; Hobbs and Thorburn, 1997; Lemoine et al., 1997; Smith et al., 1998; Cayrel et al., 1999); we have now only upper limits on the elemental ratio, $\mathrm{Li} / \mathrm{H}$, and the isotopic ratio, ${ }^{6} \mathrm{Li} /{ }^{7} \mathrm{Li}$ (in number):

$$
\mathrm{Li} / \mathrm{H} \lesssim 1 \times 10^{-9} \quad \text { and } \quad{ }^{6} \mathrm{Li} /{ }^{7} \mathrm{Li} \leq 0.1 \text {. }
$$

It should be noted that there exist Li-deficient stars of which $\mathrm{Li}$ abundance is lower than the above by one or two orders of magnitude (e.g., Soderblom et al., 1993). It is believed that in such stars the surface materials are transported to a hot region by a large scale mixing and, as a result, light elements such as ${ }^{2} \mathrm{H},{ }^{6} \mathrm{Li}$, and ${ }^{7} \mathrm{Li}$ are decomposed.

The $\mathrm{Be} / \mathrm{Li}$ ratio is evaluated from observations of stars of which surface temperature is higher than $6700 \mathrm{~K}$; Meneguzzi et al. (1971) gave the ratio of $\mathrm{Be} / \mathrm{Li}$ between $2 \times 10^{-3}$ and $1.5 \times 10^{-2}$ (in number) from the observations of eight mainsequence stars with masses between $1 M_{\odot}$ and $2 M_{\odot}$. Furthermore, Boesgaard and Budge (1989) reported $4.7 \times 10^{-3}$ and $5.0 \times 10^{-2}$ for two Li-deficient F-type stars in Hyades. Since ${ }^{6} \mathrm{Li} /{ }^{7} \mathrm{Li} \lesssim 0.1$, the probable range of ${ }^{9} \mathrm{Be} /{ }^{7} \mathrm{Li}$ in the presupernova stage may be given as (in number)

$$
2 \times 10^{-3} \lesssim{ }^{9} \mathrm{Be} /{ }^{7} \mathrm{Li} \lesssim 5 \times 10^{-2} .
$$

As will be seen later, the above abundance of ${ }^{9} \mathrm{Be}$ is one or two orders of magnitude larger than that produced in the exploding H-rich envelope.

There are also many observational studies on the $\mathrm{B} / \mathrm{Li}$ ratio of stars. Lemke et al. (1993) reported the $\mathrm{B} / \mathrm{Li}$ ratio to be $2.9 \times 10^{-2}$ and $1.4 \times 10^{-2}$ (in number) from the observations of HD82328 and HD211027, respectively, both of which are population I stars with the surface temperature higher than $6000 \mathrm{~K}$. Furthermore, Boesgaard et al. (1998) found $2.1 \times 10^{-1}$ and 1.0 in $\mathrm{Li}$ - and Be-deficient two stars. On the basis of these observational studies, the probable range of the ${ }^{11} \mathrm{~B} /{ }^{7} \mathrm{Li}$ ratio is evaluated as

$$
1 \times 10^{-2} \lesssim{ }^{11} \mathrm{~B} /{ }^{7} \mathrm{Li} \lesssim 1
$$

\section{Method of Calculation}

\subsection{Model of supernova explosion}

In the present study we employ the same model of the supernova explosion and the same nuclear reaction network as in Paper I. One of the important preparations for pursuing the explosive nucleosynthesis is to know time variations of the temperature and the density inside the supernova, which 
Table 1. Probable initial mass fractions and isotopic ratios (in number) of the CNO-elements in the H-rich envelope. For comparison, we also present the chemical compositions given by Woosley et al. (1990) and Weaver and Woosley (1993) (referred to as W90 and WW93, respectively) as well as the steady-flow equilibrium compositions of the CNO-cycle.

\begin{tabular}{c|c|ccc}
\hline & Probable values & W90 & WW93 & Equilibrium values \\
\hline${ }^{4} \mathrm{He}$ & $0.25 \sim 0.6$ & $\sim 0.5$ & 0.39 & $0.25 \sim 0.60$ \\
${ }^{12} \mathrm{C}$ & $2 \times 10^{-4} \sim 2 \times 10^{-3}$ & $2.1 \times 10^{-4}$ & $2 \times 10^{-3}$ & $2.2 \times 10^{-4} \sim 4.4 \times 10^{-4}$ \\
${ }^{14} \mathrm{~N}$ & $5 \times 10^{-3} \sim 2 \times 10^{-2}$ & $1.2 \times 10^{-2}$ & $5 \times 10^{-3}$ & $1.9 \times 10^{-2}$ \\
${ }^{16} \mathrm{O}$ & $3 \times 10^{-4} \sim 7 \times 10^{-3}$ & $6.6 \times 10^{-4}$ & $7 \times 10^{-3}$ & $2.9 \times 10^{-4} \sim 6.3 \times 10^{-4}$ \\
\hline${ }^{12} \mathrm{C} /{ }^{4} \mathrm{He}$ & $1 \times 10^{-4} \sim 2 \times 10^{-3}$ & $1.4 \times 10^{-4}$ & $1.7 \times 10^{-3}$ & $1.2 \times 10^{-4} \sim 5.9 \times 10^{-4}$ \\
${ }^{14} \mathrm{~N} /{ }^{1} \mathrm{H}$ & $9 \times 10^{-4} \sim 5 \times 10^{-3}$ & $1.7 \times 10^{-3}$ & $9.2 \times 10^{-4}$ & $2.3 \times 10^{-3} \sim 5.4 \times 10^{-3}$ \\
${ }^{12} \mathrm{C} /{ }^{13} \mathrm{C}$ & $3 \sim 2 \times 10$ & 3.4 & $2.4 \times 10$ & 3.5 \\
${ }^{14} \mathrm{~N} /{ }^{15} \mathrm{~N}$ & $3 \times 10^{4}$ & $3.2 \times 10^{4}$ & - & $2.9 \times 10^{4} \sim 3.4 \times 10^{4}$ \\
${ }^{16} \mathrm{O} /{ }^{17} \mathrm{O}$ & $2 \times 10^{2} \sim 2 \times 10^{3}$ & - & $1.2 \times 10^{2}$ & $2.2 \times 10^{2} \sim 9.1 \times 10^{2}$ \\
\hline
\end{tabular}

Table 2. Probable initial mass fractions and isotopic ratios (in number) of the CNO-elements in the radiative He-layer. In this table, W90 and WW93 denote a composition of the ashes of hydrogen-burning in W90 and that of the radiative He-layer in WW93, respectively.

\begin{tabular}{c|c|ccc}
\hline & Probable values & W90 & WW93 & Equilibrium values \\
\hline${ }^{4} \mathrm{He}$ & $0.95 \sim 1$ & 0.985 & 0.98 & $0.95 \sim 0.97$ \\
${ }^{12} \mathrm{C}$ & $2 \times 10^{-4} \sim 4 \times 10^{-4}$ & $2.2 \times 10^{-4}$ & $2 \times 10^{-4}$ & $2.2 \times 10^{-4} \sim 4.4 \times 10^{-4}$ \\
${ }^{14} \mathrm{~N}$ & $1 \times 10^{-2} \sim 2 \times 10^{-2}$ & $1.2 \times 10^{-2}$ & $1.3 \times 10^{-2}$ & $1.9 \times 10^{-2}$ \\
${ }^{16} \mathrm{O}$ & $2 \times 10^{-4} \sim 6 \times 10^{-4}$ & $2.6 \times 10^{-4}$ & $2 \times 10^{-4}$ & $2.9 \times 10^{-4} \sim 6.3 \times 10^{-4}$ \\
\hline${ }^{12} \mathrm{C} /{ }^{4} \mathrm{He}$ & $7 \times 10^{-5} \sim 2 \times 10^{-4}$ & $7.4 \times 10^{-5}$ & $6.8 \times 10^{-5}$ & $7.6 \times 10^{-5} \sim 1.5 \times 10^{-4}$ \\
${ }^{12} \mathrm{C} /{ }^{13} \mathrm{C}$ & $3 \sim 4$ & 3.5 & 2.7 & 3.5 \\
${ }^{14} \mathrm{~N} /{ }^{15} \mathrm{~N}$ & $3 \times 10^{4}$ & $3.1 \times 10^{4}$ & - & $2.9 \times 10^{4} \sim 3.4 \times 10^{4}$ \\
${ }^{16} \mathrm{O} /{ }^{17} \mathrm{O}$ & $2 \times 10^{2} \sim 9 \times 10^{2}$ & - & $1.1 \times 10$ & $2.2 \times 10^{2} \sim 9.1 \times 10^{2}$ \\
\hline
\end{tabular}

Table 3. Probable initial mass fractions and isotopic ratios (in number) of the CNO-elements in the convective He-layer. In this table, W90 denotes a composition of the He-layer with 95\% He estimated by W90 and WW93 denotes that of the convective He-layer given in WW93.

\begin{tabular}{c|c|ccc}
\hline & Probable values & W90 & WW93 & Paper I \\
\hline${ }^{4} \mathrm{He}$ & $0.91 \sim 0.95$ & 0.95 & 0.91 & 0.93 \\
${ }^{12} \mathrm{C}$ & $3 \times 10^{-2} \sim 6 \times 10^{-2}$ & $3.0 \times 10^{-2}$ & $6 \times 10^{-2}$ & $6.2 \times 10^{-2}$ \\
${ }^{14} \mathrm{~N}$ & $1 \times 10^{-12} \sim 6 \times 10^{-8}$ & $1.1 \times 10^{-12}$ & $6 \times 10^{-7} \sim 5 \times 10^{-6}$ & $5.8 \times 10^{-8}$ \\
${ }^{16} \mathrm{O}$ & $4 \times 10^{-4} \sim 1 \times 10^{-2}$ & $4.1 \times 10^{-4}$ & $1.1 \times 10^{-2}$ & $7.2 \times 10^{-4}$ \\
${ }^{18} \mathrm{O}$ & $1 \times 10^{-5}$ & $8.0 \times 10^{-3}$ & $2.0 \times 10^{-3}$ & $1.4 \times 10^{-5}$ \\
\hline${ }^{12} \mathrm{C} /{ }^{4} \mathrm{He}$ & $1 \times 10^{-2} \sim 2 \times 10^{-2}$ & $1.1 \times 10^{-2}$ & $2.2 \times 10^{-2}$ & $2.2 \times 10^{-2}$ \\
${ }^{12} \mathrm{C} /{ }^{16} \mathrm{O}$ & $7 \sim 1 \times 10^{2}$ & $9.8 \times 10$ & 7.3 & $1.1 \times 10^{2}$ \\
${ }^{12} \mathrm{C} /{ }^{13} \mathrm{C}$ & $9 \times 10^{7} \sim 2 \times 10^{9}$ & $9.3 \times 10^{7}$ & - & $2.3 \times 10^{9}$ \\
${ }^{14} \mathrm{~N} /{ }^{15} \mathrm{~N}$ & $4 \times 10^{-5} \sim 4$ & $3.7 \times 10^{-5}$ & - & 4.0 \\
${ }^{16} \mathrm{O} /{ }^{17} \mathrm{O}$ & $5 \times 10^{4} \sim 8 \times 10^{4}$ & - & $5.8 \times 10^{3}$ & $5.5 \times 10^{4}$ \\
${ }^{16} \mathrm{O} /{ }^{18} \mathrm{O}$ & $5 \times 10^{1} \sim 1 \times 10^{3}$ & $5.8 \times 10^{-2}$ & 6.2 & $5.8 \times 10^{1}$ \\
\hline
\end{tabular}

are caused by a shock heating and a subsequent expansion. In Paper I, as a shock propagation model, we employed the generalized Sedov solution of a spherically symmetric strong shock wave in a medium with a power law initial density profile (Sedov, 1959). The power law index of the density profile of the presupernova was chosen so as to fit $14 \mathrm{E} 1$ model (i.e., the presupernova model of a $16.2 M_{\odot}$ star, which corresponds to a $20 M_{\odot}$ main sequence star, after mass loss process) given by Shigeyama and Nomoto (1990).

Our nuclear reaction network, the main tool for simulating the explosive nucleosynthesis of the $X$-elements and the CNO-elements, was constructed from 52 species of isotopes from ${ }^{1} \mathrm{H}$ to ${ }^{23} \mathrm{Mg}$. All nuclear reactions in this network were compiled in Yoshida (2000). Neutrino-induced reactions (Woosley et al., 1990) were also included in our network and their reaction rates were taken from Hoffman and Woosley (1992). As shown in Paper I, the decay time of the neutrino flux, $\tau_{v}$, and the total energy carried by neutrinos, $E_{v}$, are important parameters governing the role of neutrino-induced reactions on the production of the $X$-elements. In this study, they are fixed commonly to be

$$
\tau_{v}=3 \quad \mathrm{~s} \quad \text { and } \quad E_{v}=3 \times 10^{53} \mathrm{erg},
$$

which are the same as those of "the standard model" in Paper I. The temperature of $i$-flavor neutrino, $T_{v i}$, is also fixed 
Table 4. Initial chemical compositions of the 4-component models and model SI (by mass fraction). In all models except model SI, the mass fractions of species other than tabulated ones (including ${ }^{1} \mathrm{H}$ in the He-layer) are set to be zero. In model SI, ${ }^{14} \mathrm{~N}$ and the other rare light elements are also included. The mass fractions are as follows: ${ }^{1} \mathrm{H}=0$ (conv. He) and $1.0 \times 10^{-5}(\mathrm{rad} . \mathrm{He}),{ }^{14} \mathrm{~N}=5.8 \times 10^{-8}$ (conv. He) and $4.8 \times 10^{-3}$ (rad. He and $\mathrm{H}-\mathrm{env}$.), the $X$-elements $\leq 1.0 \times 10^{-10}$, and the rare CNO-elements $\leq 3.0 \times 10^{-5}$.

\begin{tabular}{|c|c|c|c|c|c|c|c|c|c|c|}
\hline \multirow{2}{*}{ Model } & \multicolumn{4}{|c|}{ H-rich Envelope } & \multicolumn{3}{|c|}{ Radiative He-layer } & \multicolumn{3}{|c|}{ Convective He-layer } \\
\hline & ${ }^{1} \mathrm{H}$ & ${ }^{4} \mathrm{He}$ & ${ }^{12} \mathrm{C}$ & ${ }^{16} \mathrm{O}$ & ${ }^{4} \mathrm{He}$ & ${ }^{12} \mathrm{C}$ & ${ }^{16} \mathrm{O}$ & ${ }^{4} \mathrm{He}$ & ${ }^{12} \mathrm{C}$ & ${ }^{16} \mathrm{O}$ \\
\hline SII & 0.5500 & 0.4300 & 0.0100 & 0.0100 & 0.9800 & 0.0100 & 0.0100 & 0.9372 & 0.0314 & 0.0314 \\
\hline A1 & \multirow{2}{*}{0.5500} & \multirow{2}{*}{0.4300} & 0.0190 & 0.0010 & \multirow{4}{*}{0.9800} & 0.0190 & 0.0010 & \multirow{2}{*}{0.9372} & 0.0622 & 0.0006 \\
\hline $\mathrm{A} 2$ & & & 0.0010 & 0.0190 & & 0.0010 & 0.0190 & & 0.0006 & 0.0622 \\
\hline $\mathrm{A} 3$ & 0.7300 & 0.2500 & \multirow{2}{*}{0.0100} & \multirow{2}{*}{0.0100} & & \multirow{2}{*}{0.0100} & \multirow{2}{*}{0.0100} & 0.8000 & 0.1000 & 0.1000 \\
\hline A4 & 0.2500 & 0.7300 & & & & & & 0.5000 & 0.2500 & 0.2500 \\
\hline A5 & \multirow{2}{*}{0.5500} & 0.4480 & 0.0010 & 0.0010 & 0.9980 & 0.0010 & 0.0010 & \multirow{2}{*}{0.9372} & \multirow{2}{*}{0.0314} & \multirow{2}{*}{0.0314} \\
\hline A6 & & 0.4498 & 0.0001 & 0.0001 & 0.9998 & 0.0001 & 0.0001 & & & \\
\hline SI & 0.5650 & 0.4300 & $8.5 \times 10^{-5}$ & 0.0001 & 0.9950 & $8.5 \times 10^{-5}$ & 0.0001 & 0.9300 & 0.0611 & 0.0007 \\
\hline
\end{tabular}

to be the same as that in Paper I:

$$
T_{\nu e}=T_{\bar{\nu} e}=4 \mathrm{MeV} / k
$$

and

$$
T_{\nu \mu}=T_{\bar{\nu} \mu}=T_{\nu \tau}=T_{\bar{\nu} \tau}=8 \mathrm{MeV} / k,
$$

where $k$ is the Boltzmann constant.

\subsection{Initial chemical compositions}

3.2.1 Four-component models In Paper I we showed that the $X$-elements and most of the CNO-elements are mainly produced from ${ }^{4} \mathrm{He},{ }^{12} \mathrm{C}$, and ${ }^{16} \mathrm{O}$ through neutrino-induced reactions. So it is expected, at least qualitatively, that the production of the $X$-elements and the $\mathrm{CNO}$-elements essentially depends on the amounts of ${ }^{4} \mathrm{He},{ }^{12} \mathrm{C}$, and ${ }^{16} \mathrm{O}$ in the presupernova stage. However, it is difficult to estimate quantitatively from the results of Paper I how the abundance ratios of the light elements are changed by the adopted initial chemical compositions. In order to answer such a question, we set artificially 4-component models of the initial chemical compositions which consist of only ${ }^{1} \mathrm{H},{ }^{4} \mathrm{He},{ }^{12} \mathrm{C}$, and ${ }^{16} \mathrm{O}$. The amounts of the other elements included in our nuclear reaction network are put practically to be zero. Note that in the above models we omit ${ }^{14} \mathrm{~N}$ completely from the initial constituents in order to see clearly the formation processes of ${ }^{14} \mathrm{~N}$ and ${ }^{15} \mathrm{~N}$ although ${ }^{14} \mathrm{~N}$ is the most abundant element among the CNO-elements in the radiative He-layer and the H-rich envelope. The effects of this simplification will be discussed in the later sections where we will see such a simplification does not affect the main conclusions of the present study.

At first, we will consider the following initial chemical composition as a standard model of this study. In the convective and the radiative He-layers, the mass fractions of ${ }^{4} \mathrm{He}$ are taken to be 0.9372 and 0.9800 , respectively. The remainder is divided into ${ }^{12} \mathrm{C}$ and ${ }^{16} \mathrm{O}$ equally by weight (their mass fractions are 0.0314 and 0.0100 , respectively, in the convective and the radiative $\mathrm{He}$-layers). In the $\mathrm{H}$-rich envelope, the mass fractions of ${ }^{1} \mathrm{H}$ and ${ }^{4} \mathrm{He}$ are set to be 0.55 and 0.43 , respectively, the values of which are almost equal to those of Paper I. Note that in this model the initial metallicity is equal to 0.02 . Hereafter, this model will be called model SII (the standard model of the present study) and the chemical composition adopted in Paper I will be called that of model SI.

In order to investigate in detail the influence of the initial chemical composition on the light element synthesis, we prepare additional six models of the initial chemical compositions other than models SI and SII. They are tabulated in Table 4 together with models SI and SII. Models A1 and A2 are of seeing the role of ${ }^{12} \mathrm{C}$ and ${ }^{16} \mathrm{O}$ separately on the light element synthesis and correspond to $\mathrm{C}$-rich and O-rich compositions, respectively. In models A3 and A4, we see the influence of a variety of $\mathrm{He}$ abundances. In these models, taking account of the depletion of ${ }^{4} \mathrm{He}$ due to the He-shell burning, we adopt less abundant ${ }^{4} \mathrm{He}$-content (i.e., 0.8 and 0.5 in models A3 and A4, respectively) in the convective Helayer but the same chemical composition as in model SII in the radiative He-layer. Furthermore, in the H-rich envelope, in model A3 the mass fractions of ${ }^{1} \mathrm{H}$ and ${ }^{4} \mathrm{He}$ are taken to be 0.73 and 0.25 (almost the same as the solar-system abundances) whereas in model A4 the mass fractions of ${ }^{1} \mathrm{H}$ and ${ }^{4} \mathrm{He}$ are set to be 0.25 and 0.73 , respectively. In all cases mentioned above, the metallicity (i.e., the total mass fractions of ${ }^{12} \mathrm{C}$ and ${ }^{16} \mathrm{O}$ in our present study) is put to be the same value as in model SII. In models A5 and A6 we consider the effect due to a variety of the metallicities. In models A5 and A6, the metallicity is set to be 0.002 and 0.0002 , respectively. In both cases the metal component consists of ${ }^{12} \mathrm{C}$ and ${ }^{16} \mathrm{O}$ with equal mass fraction. The mass fractions of ${ }^{1} \mathrm{H}$ and ${ }^{4} \mathrm{He}$ are taken to be nearly equal to those of model SII.

It should be noted that, in some cases, the model compositions are far extremely from those expected properly in the presupernova stage: in the convective He-layer, models A2 and A4 give extraordinarily large ${ }^{16} \mathrm{O} /{ }^{12} \mathrm{C}$ ratio and too much metallicity, respectively. Furthermore, in model A1 the ${ }^{16} \mathrm{O} /{ }^{12} \mathrm{C}$ ratio is too low in the radiative He-layer and the $\mathrm{H}$-rich envelope. So, we will exclude these models when we discuss the probable elemental/isotopic ratios of supernova ejecta in Section 5.

3.2.2 Over-abundant model of rare light elements In the models mentioned above, we neglect completely the initial abundances of the $X$-elements and the rare CNOelements. We are interested in, not only the production of the 
Table 5. Typical key reactions of light element synthesis in model SII. The upper and lower columns show the formation and decomposition reactions, respectively. Empty columns mean that there are no appreciable formation (or decomposition) processes.

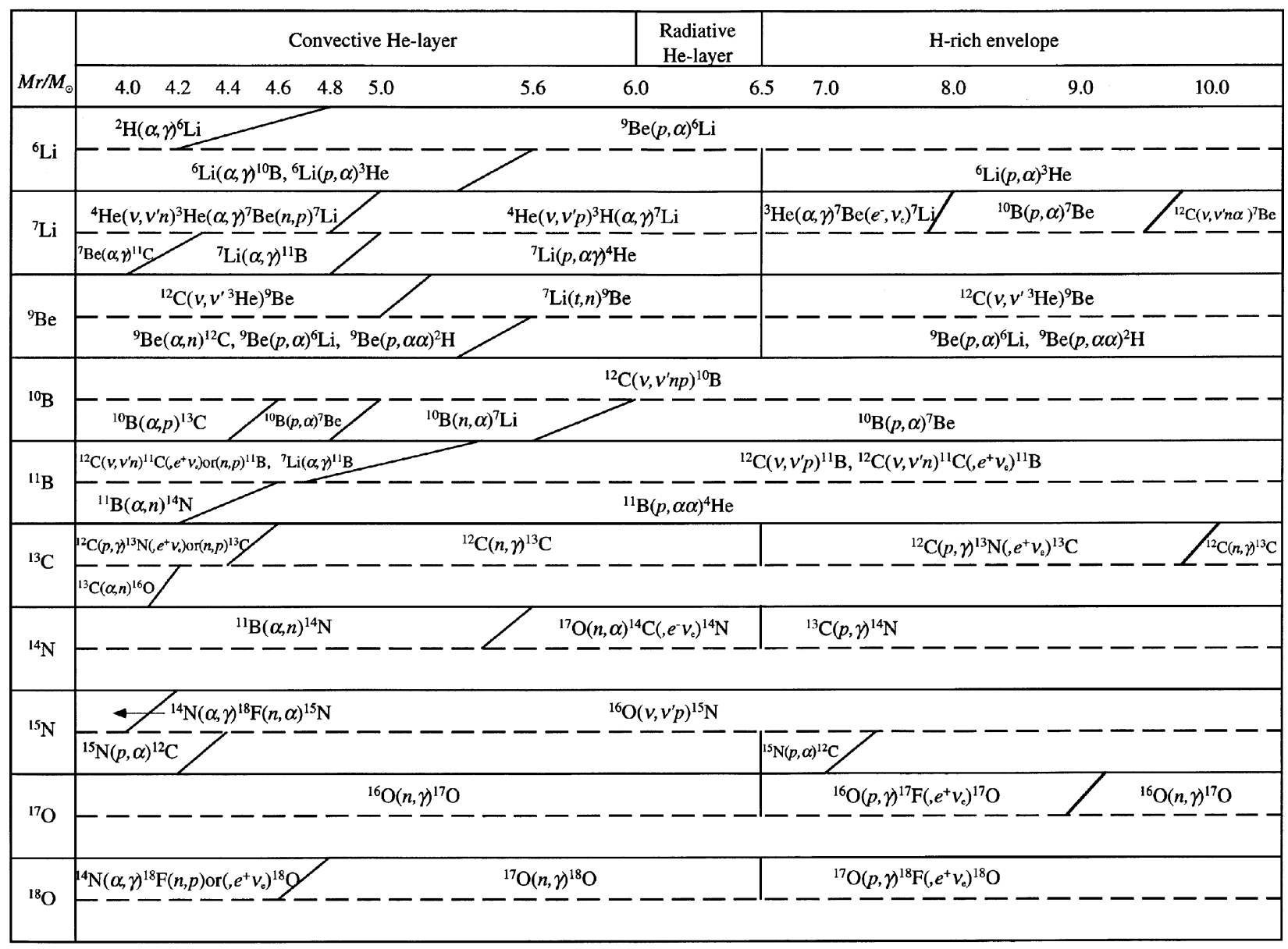

light elements from ${ }^{4} \mathrm{He},{ }^{12} \mathrm{C}$, and ${ }^{16} \mathrm{O}$, but also the destruction of these rare elements which have been formed through other processes and existed just before the supernova explosion. So we make a simulation in the alternative and artificial case where the initial amounts of the $X$-elements and the rare CNO-elements are set to be $1 \times 10^{-10}$ and $1 \times 10^{-6}$, respectively, in all mass coordinates (such a model is called "model B"). The four major components, i.e., ${ }^{1} \mathrm{H},{ }^{4} \mathrm{He},{ }^{12} \mathrm{C}$, and ${ }^{16} \mathrm{O}$, are fixed to be the same as in model SII.

\section{Numerical Results}

\subsection{General features of the light element production}

At first, we will see, in brief, the general features of element synthesis due to the 4-component model, comparing the produced amounts of the light elements between models SI and SII. The initial chemical compositions of main constituents of models SI and SII are shown in Table 4; in short, differences among the two models are summarized as (1) in the convective He-layer ${ }^{12} \mathrm{C} /{ }^{16} \mathrm{O}$ (by weight) are equal to 1 and about 100 in models SII and SI, respectively, (2) in the radiative He-layer and the H-rich envelope, the mass fractions of ${ }^{12} \mathrm{C}$ and ${ }^{16} \mathrm{O}$ in model SII are about 100 times as large as those of model SI, and (3) there exist initially no $X$-elements and rare CNO-elements in model SII.

Distributions of the mass fractions of the $X$-elements and the CNO-elements are presented in Figs. 1(a) and (b), re- spectively. Main production and decomposition processes of the $X$-elements and the CNO-elements in model SII are tabulated in Table 5. The comparison between models SI and SII suggests the following interesting points which are important to our later arguments. That is,

(1) Roughly speaking, the light elements are classified into three groups, i.e., (a) ${ }^{6} \mathrm{Li},{ }^{7} \mathrm{Li},{ }^{9} \mathrm{Be},{ }^{14} \mathrm{~N}$, and ${ }^{18} \mathrm{O}$, (b) ${ }^{10} \mathrm{~B},{ }^{11} \mathrm{~B}$, and ${ }^{13} \mathrm{C}$, and $(\mathrm{c}){ }^{15} \mathrm{~N}$ and ${ }^{17} \mathrm{O}$. The produced amounts of elements of groups (a), (b), and (c) are mainly determined by the contents of ${ }^{4} \mathrm{He},{ }^{12} \mathrm{C}$, and ${ }^{16} \mathrm{O}$, respectively.

(2) About ${ }^{10} \mathrm{~B}$ and ${ }^{15} \mathrm{~N}$, another production channel opens possibly if there exist appreciable amounts of ${ }^{14} \mathrm{~N}$ and ${ }^{18} \mathrm{O}$ in the presupernova stage, respectively (see also Section 5.4 for ${ }^{15} \mathrm{~N}$ ).

(3) In the radiative He-layer and the H-rich envelope, the final abundances of the CNO-elements are essentially governed by the initial chemical composition in a sense that pre-existing elements survive as they were.

Hereafter we will compare the results of the two models more precisely and we will confirm the above conjectures in the later subsections.

In the convective He-layer, the amounts of the $X$-elements in model SII are very similar to those in model SI; the final 
abundances of ${ }^{6} \mathrm{Li},{ }^{7} \mathrm{Li}$, and ${ }^{9} \mathrm{Be}$ are quite the same between the two models as long as the mass fractions are larger than the level of $1 \times 10^{-14}$. For ${ }^{10} \mathrm{~B}$ and ${ }^{11} \mathrm{~B}$, the mass fractions are lower than those in model SI by a factor of about two, in accordance with the abundance ratio of ${ }^{12} \mathrm{C}$ between the two models.

In the H-rich envelope, the amounts of ${ }^{6} \mathrm{Li},{ }^{7} \mathrm{Li}$, and ${ }^{9} \mathrm{Be}$ in model SII are also similar to those in model SI except the ${ }^{7} \mathrm{Li}$-abundance in the outer region (i.e., $M_{r} \gtrsim 9.0 M_{\odot}$ ) which, in the case of model SI, is mainly governed by the initial abundance (the initial amount of ${ }^{7} \mathrm{Li}$ is about $2 \times 10^{-11}$ in model SI). The amount of ${ }^{11} \mathrm{~B}$ is remarkably different among the two models: the abundance in model SII is two orders of magnitude larger than that in model SI (also in the radiative He-layer). The large amount of ${ }^{12} \mathrm{C}$ in model SII, about two orders of magnitude larger than that in model SI, brings about this difference (note that ${ }^{11} \mathrm{~B}$ is commonly formed from ${ }^{12} \mathrm{C}$ through the $v$-processes in both models). It is interesting to note that, although the amounts of ${ }^{10} \mathrm{~B}$ are quite similar between models SI and SII, the production process is different. In the case of model SII, ${ }^{10} \mathrm{~B}$ is produced through ${ }^{12} \mathrm{C}\left(v, v^{\prime} n p\right){ }^{10} \mathrm{~B}$ because of the large amount of ${ }^{12} \mathrm{C}$ and no initial amount of ${ }^{14} \mathrm{~N}$. In the case of model $\mathrm{SI},{ }^{10} \mathrm{~B}$ is produced through ${ }^{14} \mathrm{~N}\left(v, v^{\prime} \alpha\right){ }^{10} \mathrm{~B}$ since ${ }^{14} \mathrm{~N}$ is abundant.

In the radiative He-layer, the abundances of ${ }^{6} \mathrm{Li}$ and ${ }^{9} \mathrm{Be}$
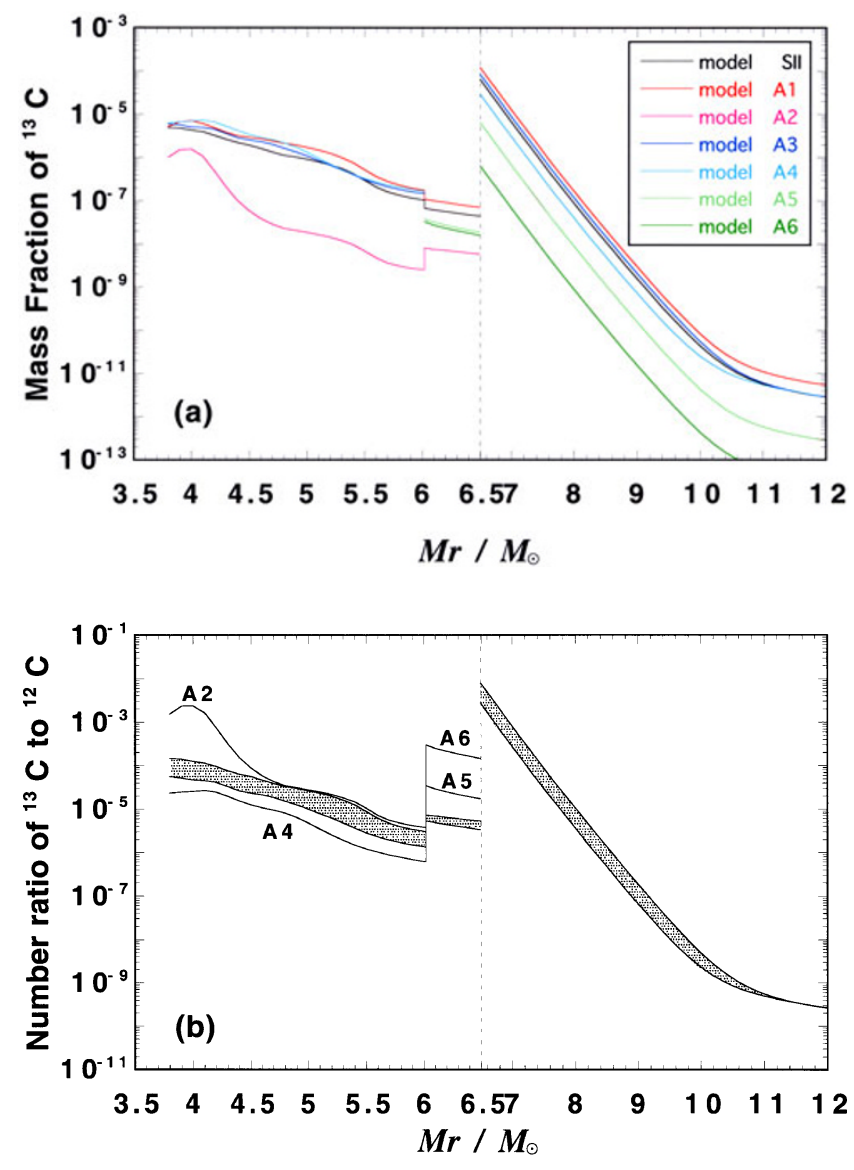

Fig. 2. Distributions of the final mass fraction of ${ }^{13} \mathrm{C}$ (panel(a)) and the number ratio of ${ }^{13} \mathrm{C}$ to ${ }^{12} \mathrm{C}$ (panel(b)). In panel (a), some lines overlap each other: models SII, A5, and A6 in the convective He-layer and models SII, A3, and A4 in the radiative He-layer. In panel (b), the shaded regions show ${ }^{13} \mathrm{C} /{ }^{12} \mathrm{C}$ ratio in most models which give the similar results. Exceptional curves are labeled model names. are different between the two models in contrast to the case of the H-rich envelope; the difference comes from the fact that, in model SII, they are scarcely decomposed because there are no protons initially (see table 3 in Paper I). The abundance behavior of the other $X$-elements are similar to those in the H-rich envelope.

Distributions of the amounts of the CNO-elements are different between models SI and SII. Difference in ${ }^{12} \mathrm{C}$ - and ${ }^{16} \mathrm{O}$-abundances observed in all regions is due, simply, to the adopted initial abundances because, as shown in Paper I, they are neither formed nor decomposed appreciably during the supernova explosion. In the convective He-layer, the final abundances of ${ }^{13} \mathrm{C}$ and ${ }^{15} \mathrm{~N}$ are similar, within one order of magnitude or so, to those of model SI. The initial abundances of these isotopes adopted in model SI are below the level of $1 \times 10^{-8}$ which is comparable to or lower than that of the explosive production and, hence, we cannot see an appreciable difference between the two models. The distribution of the ${ }^{17} \mathrm{O}$ abundance behaves also similarly to that in Paper I but the abundance strongly reflects that of ${ }^{16} \mathrm{O}$. On the other hand, for ${ }^{14} \mathrm{~N}$ and ${ }^{18} \mathrm{O}$ the abundance patterns are quite different; this is due, mainly, to the fact that the initial abundances govern essentially the final abundances in model SI. In the case of model SII, the abundances in the innermost region are comparable to those of model SI but decrease abruptly
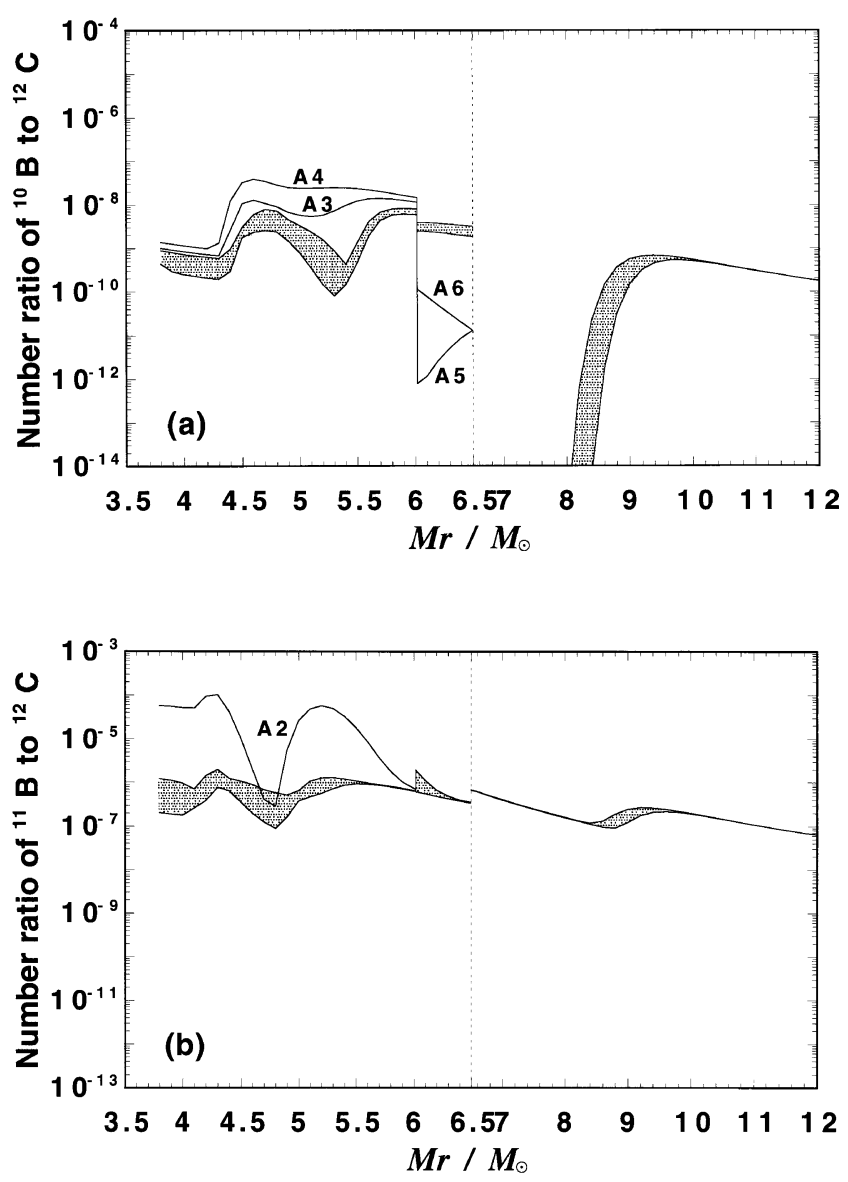

Fig. 3. Distributions of the number ratio of ${ }^{10} \mathrm{~B}$ to ${ }^{12} \mathrm{C}$ (panel(a)) and that of ${ }^{11} \mathrm{~B}$ to ${ }^{12} \mathrm{C}$ (panel(b)). The shaded regions show the ratios in most models which give the similar results. In the exceptional cases, we show curves independently. 
with an increase in $M_{r}$. This behavior is brought about by large temperature dependence of their $\alpha$-capture production processes (see Table 5).

In the radiative He-layer and the H-rich envelope, the abundance patterns of the rare CNO-elements are different between the two models, SI and SII. This is because, in model SI, these elements are scarcely produced during the supernova explosion and their final abundances are almost determined by their initial abundances. In the H-rich envelope, the CNO-elements are produced only in the inner region. The maximum amounts of ${ }^{13} \mathrm{C}$ and ${ }^{17} \mathrm{O}$, of which seed nuclei are ${ }^{12} \mathrm{C}$ and ${ }^{16} \mathrm{O}$, respectively, are larger than that in model SI. This is simply because the amounts of ${ }^{12} \mathrm{C}$ and ${ }^{16} \mathrm{O}$ are larger than those in model SI by a factor of 100 . Heavy nitrogen, ${ }^{15} \mathrm{~N}$, is produced from ${ }^{16} \mathrm{O}$ through the $v$ process in this case but from ${ }^{14} \mathrm{~N}$ in model SI, so that the abundance pattern differs from each other. In model SII, ${ }^{14} \mathrm{~N}$ and ${ }^{18} \mathrm{O}$ are hardly produced because their parent nuclei, ${ }^{13} \mathrm{C}$ and ${ }^{17} \mathrm{O}$, are much less compared with the case of model SI.

\subsection{Sensitivity to the initial compositions}

4.2.1 ${ }^{13} \mathrm{C} \quad$ As an example, we will first see how the amount of ${ }^{13} \mathrm{C}$ depends on the adopted abundances of the major four components. The distributions of the final mass fractions of ${ }^{13} \mathrm{C}$ are illustrated in Fig. 2(a) for all models. In most part of the convective He-layer, we see the similar distributions of the final ${ }^{13} \mathrm{C}$ in all models other than model $\mathrm{A} 2$. In the case of model $\mathrm{A} 2$, the amount of ${ }^{13} \mathrm{C}$ is smaller than that of the others by a factor of 40. As seen from Table 5, ${ }^{13} \mathrm{C}$ is formed through $(n, \gamma)$-reaction of ${ }^{12} \mathrm{C}$. Almost all of neutrons are produced from ${ }^{4} \mathrm{He}$ through the $\nu$-process and consumed by ${ }^{12} \mathrm{C}$ and ${ }^{16} \mathrm{O}$ with a similar probability per nucleus in the temperature range of the convective He-layer. Hence, the amount of ${ }^{13} \mathrm{C}$ is nearly proportional to the number ratio of ${ }^{12} \mathrm{C}$ to ${ }^{12} \mathrm{C}+{ }^{16} \mathrm{O}$. In model $\mathrm{A} 2$ where the ratio of ${ }^{12} \mathrm{C} /\left({ }^{12} \mathrm{C}+{ }^{16} \mathrm{O}\right)$ is very small compared with those of the other models, the resultant abundance of ${ }^{13} \mathrm{C}$ is very small.

In the radiative He-layer, the maximum and minimum amounts of ${ }^{13} \mathrm{C}$ are seen in models $\mathrm{A} 1$ and $\mathrm{A} 2$, respectively. Among models SII, A1, and A2, the total amount of ${ }^{12} \mathrm{C}$ and ${ }^{16} \mathrm{O}$ is identical, so that the production of ${ }^{13} \mathrm{C}$ is governed by the ${ }^{12} \mathrm{C} /{ }^{16} \mathrm{O}$ ratio, as in the case of the convective He-layer, i.e., the large ${ }^{12} \mathrm{C} /{ }^{16} \mathrm{O}$ ratio brings out the large amount of ${ }^{13} \mathrm{C}$, and vice versa. In models $\mathrm{A} 5$ and $\mathrm{A} 6$, an appreciable amount of ${ }^{13} \mathrm{C}$ is produced in spite of the low metallicity because of the small abundances of neutron poisons, ${ }^{12} \mathrm{C}$ and ${ }^{16} \mathrm{O}$. In the $\mathrm{H}$-rich envelope, ${ }^{13} \mathrm{C}$ is simply produced through ${ }^{12} \mathrm{C}(p, \gamma){ }^{13} \mathrm{~N}\left(, e^{+} v_{\mathrm{e}}\right){ }^{13} \mathrm{C}$ and its amount is roughly proportional to those of ${ }^{12} \mathrm{C}$ and protons. So, the largest amount of ${ }^{13} \mathrm{C}$ is formed in model A1 and the smallest in model A6.

In Fig. 2(b), we show the distribution of the number of ${ }^{13} \mathrm{C}$ normalized by that of its parent isotope, ${ }^{12} \mathrm{C}$. The shaded regions show the ${ }^{13} \mathrm{C} /{ }^{12} \mathrm{C}$ ratio in most models which give the similar results, whereas the curves present those of the exceptional cases (the following figures are also illustrated by the same manner). Note that the widths of the shaded regions are confined within a factor of 3 or so. This means that the amount of ${ }^{13} \mathrm{C}$ is almost scaled by that of ${ }^{12} \mathrm{C}$ as conjectured in the first item in the second paragraph of Section 4.1. It should be also noted that the ratios, ${ }^{13} \mathrm{C} /{ }^{4} \mathrm{He}$ and ${ }^{13} \mathrm{C} /{ }^{16} \mathrm{O}$, scatter widely from two to four orders of magnitude. In model A2 where the ${ }^{12} \mathrm{C}$ content is as low as 0.0006 in the convective He-layer, the ${ }^{13} \mathrm{C} /{ }^{12} \mathrm{C}$ ratio becomes large in the innermost region. In the radiative He-layer, ${ }^{13} \mathrm{C} /{ }^{12} \mathrm{C}$ ratio is larger by a factor of 3 to 30 in models $\mathrm{A} 5$ and $\mathrm{A} 6$. The reasons are mentioned earlier. In the $\mathrm{H}$-rich envelope, ${ }^{13} \mathrm{C} /{ }^{12} \mathrm{C}$ ratio scarcely depends on initial chemical composition among all models; only difference attributes to the amount of protons and is only within a factor of $3(\simeq 0.73 / 0.25)$.

4.2.2 ${ }^{10} \mathrm{~B}$ and ${ }^{11} \mathrm{~B} \quad$ The amounts of boron isotopes are also scaled well by that of ${ }^{12} \mathrm{C}$. The distributions of ${ }^{10} \mathrm{~B} /{ }^{12} \mathrm{C}$ and ${ }^{11} \mathrm{~B} /{ }^{12} \mathrm{C}$ are presented in Figs. 3(a) and (b), respectively. We see that both ratios are insensitive to the initial chemical compositions as in the case of ${ }^{13} \mathrm{C} /{ }^{12} \mathrm{C}$ ratio except a few models.

In the He-layer, the band width of the ${ }^{10} \mathrm{~B} /{ }^{12} \mathrm{C}$ ratio is small (within one order of magnitude). This is due to the fact that ${ }^{10} \mathrm{~B}$ is mainly produced through ${ }^{12} \mathrm{C}\left(v, v^{\prime} n p\right){ }^{10} \mathrm{~B}$ and decomposed by the neutrons with the similar number density. In models A3 and A4 where both ${ }^{12} \mathrm{C}$ and ${ }^{16} \mathrm{O}$, which are poison to neutrons, are very abundant in the convective He-layer, ${ }^{10} \mathrm{~B}$ decomposition is less effective and the resultant ${ }^{10} \mathrm{~B} /{ }^{12} \mathrm{C}$ ratio becomes large. On the contrary, ${ }^{10} \mathrm{~B}$ is decomposed by neutrons more effectively in the radiative He-layer in the case of the low metallicity models (models A5 and A6). In the $\mathrm{H}$-rich envelope, ${ }^{10} \mathrm{~B}$ is decomposed completely by protons in the inner region but not in the outer region $\left(M_{r} \gtrsim 10 M_{\odot}\right)$. Hence, the abundance of ${ }^{10} \mathrm{~B}$ is determined by that of ${ }^{12} \mathrm{C}$.

In the case of ${ }^{11} \mathrm{~B}$, the behavior is somewhat different from the case of ${ }^{10} \mathrm{~B}$. In the H-rich envelope, there is no difference from model to model. In the inner part of the H-rich envelope, ${ }^{11} \mathrm{~B}$ remains as the daughter isotope of ${ }^{11} \mathrm{C}$ produced from ${ }^{12} \mathrm{C}\left(v, v^{\prime} n\right){ }^{11} \mathrm{C}$ and in the outer part ${ }^{11} \mathrm{~B}$ is produced directly from ${ }^{12} \mathrm{C}$ through the $\nu$-process. In the He-layer, even if ${ }^{12} \mathrm{C}$ and ${ }^{16} \mathrm{O}$ are very rich (models $\mathrm{A} 3$ and $\mathrm{A} 4$ ), the effect is small because ${ }^{11} \mathrm{~B}$ and ${ }^{11} \mathrm{C}$ are not so decomposed by neutrons. The variety of the ratios is within a factor of about 6. Only in the case of model $\mathrm{A} 2$ where ${ }^{12} \mathrm{C}$ is less abundant in the convective He-layer, we see a large excess of the ${ }^{11} \mathrm{~B} /{ }^{12} \mathrm{C}$ ratio. This is due to the fact that, since the abundance of ${ }^{12} \mathrm{C}$ is small, contribution of another production sequence ${ }^{3} \mathrm{H}(\alpha, \gamma){ }^{7} \mathrm{Li}(\alpha, \gamma){ }^{11} \mathrm{~B}$ becomes important. In evaluating probable ratio of ${ }^{11} \mathrm{~B} /{ }^{12} \mathrm{C}$ in Section 5.2, we will exclude the result of model A2.

4.2.3 ${ }^{15} \mathrm{~N}$ and ${ }^{17} \mathrm{O}$ Now, we consider the species of which parent isotope is ${ }^{16} \mathrm{O}$. The distribution of the number ratio of ${ }^{15} \mathrm{~N}$ to ${ }^{16} \mathrm{O}$ is presented in Fig. 4(a). Except the innermost regions of the He-layer and the H-rich envelope, ${ }^{15} \mathrm{~N} /{ }^{16} \mathrm{O}$ ratio is almost independent of the initial chemical composition. As seen from Table 5, in almost all region ${ }^{15} \mathrm{~N}$ is produced through ${ }^{16} \mathrm{O}\left(v, v^{\prime} p\right){ }^{15} \mathrm{~N}$ and is not decomposed. In the innermost regions of the He-layer and of the $\mathrm{H}$-rich envelope, the ratio of ${ }^{15} \mathrm{~N} /{ }^{16} \mathrm{O}$ depends, more or less, on the initial composition since the decomposition reaction, ${ }^{15} \mathrm{~N}(p, \alpha){ }^{12} \mathrm{C}$ becomes effective. The effect of decomposition is seen apparently for the ${ }^{12} \mathrm{C}$-poor model (model A2) because protons are mainly captured by ${ }^{12} \mathrm{C}$.

The ratio of ${ }^{17} \mathrm{O} /{ }^{16} \mathrm{O}$ is illustrated in Fig. 4(b). The ratio is confined within a factor of about 10 in the convective $\mathrm{He}$ layer except model A4. In the H-rich envelope, the ${ }^{16} \mathrm{O} /{ }^{17} \mathrm{O}$ ratio scarcely depends on the initial chemical compositions 

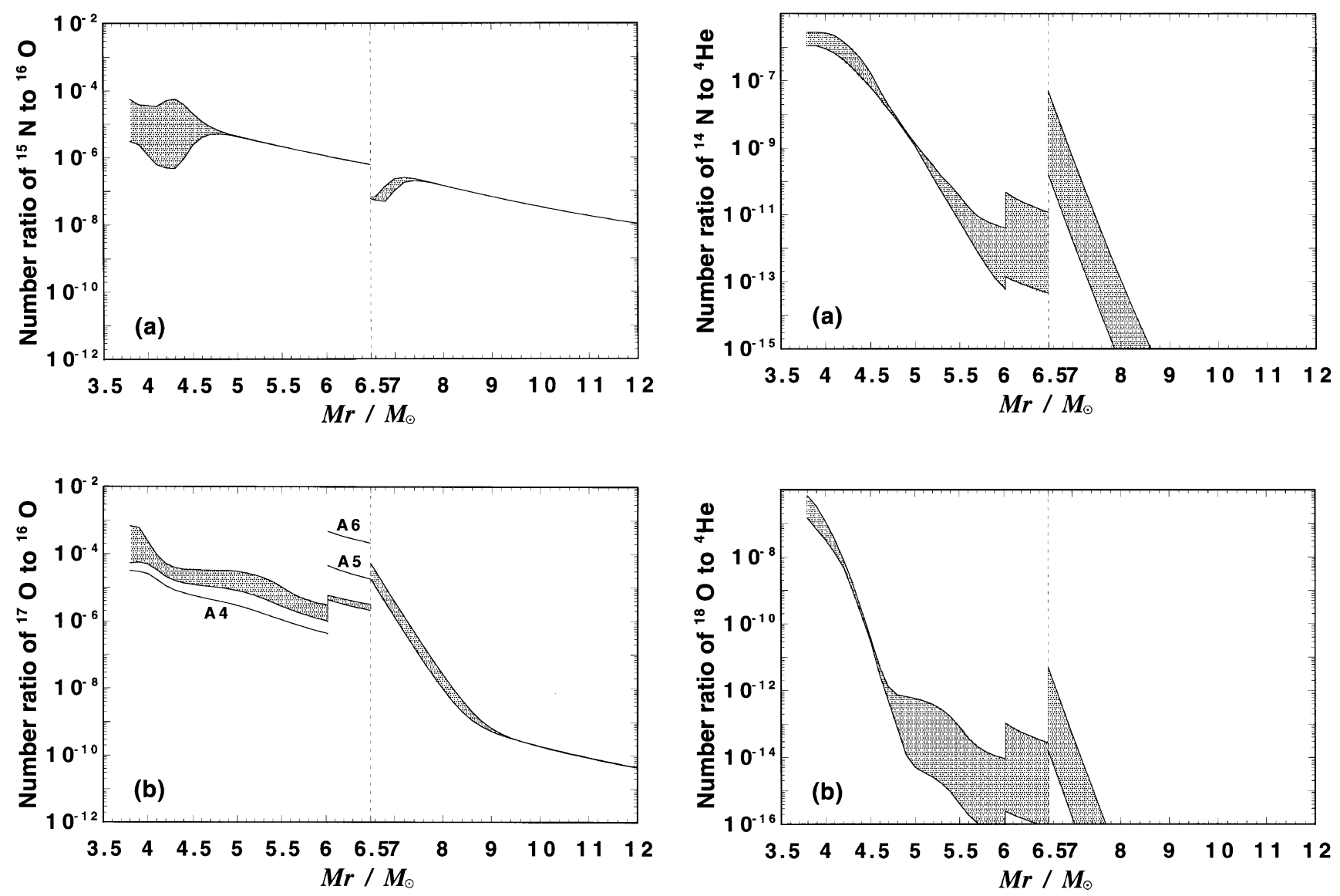

Fig. 4. As same as Fig. 3, but for the number ratio of ${ }^{15} \mathrm{~N}$ to ${ }^{16} \mathrm{O}$ (panel(a)) and that of ${ }^{17} \mathrm{O}$ to ${ }^{16} \mathrm{O}$ (panel(b))

Fig. 5. As same as Fig. 3, but for the number ratio of ${ }^{14} \mathrm{~N}$ to ${ }^{4} \mathrm{He}$ (panel(a)) and that of ${ }^{18} \mathrm{O}$ to ${ }^{4} \mathrm{He}$ (panel(b)).

since ${ }^{17} \mathrm{O}$ is produced from ${ }^{16} \mathrm{O}$ without an appreciable decomposition. The behavior of the ${ }^{17} \mathrm{O} /{ }^{16} \mathrm{O}$ ratio, shown in Fig. 4(b), is very similar to the ${ }^{13} \mathrm{C} /{ }^{12} \mathrm{C}$ ratio illustrated in Fig. 2(b) in a sense that the lack of the parent isotope brings about the high ratio. In model $\mathrm{A} 1$ where the ${ }^{16} \mathrm{O}$ content is as low as 0.0006 , the ${ }^{17} \mathrm{O} /{ }^{16} \mathrm{O}$ ratio becomes large in the innermost region of the convective He-layer (note that the upper edge of the shaded band comes from model A1). Similarly, in the radiative He-layer, the ${ }^{17} \mathrm{O} /{ }^{16} \mathrm{O}$ ratio becomes also large by a factor of 6 and of 60 in models A5 and A6 (the low metallicity models), respectively.

4.2.4 ${ }^{14} \mathrm{~N}$ and ${ }^{18} \mathrm{O}$ As seen from Table 5, both ${ }^{14} \mathrm{~N}$ and ${ }^{18} \mathrm{O}$ are produced from different parent isotopes (through different types of nuclear reactions) owing to the internal mass coordinate, $M_{r}$. So, their abundances are not always scaled thoroughly by any of the major constituents over the whole regions of $M_{r}$. Nevertheless, it is reasonable to normalize their abundances by that of ${ }^{4} \mathrm{He}$ because they are formed from ${ }^{4} \mathrm{He}$ originally with a good productivity in the innermost region of the He-layer.

The distribution of the ${ }^{14} \mathrm{~N} /{ }^{4} \mathrm{He}$ ratio is shown in Fig. 5(a). An appreciable amount of ${ }^{14} \mathrm{~N}$ is produced only in the inner region where $M_{r} \lesssim 5.0 M_{\odot}$ (see also Fig. 1(b)) and, in such a region, the ${ }^{14} \mathrm{~N} /{ }^{4} \mathrm{He}$ ratio is confined within a small region (note that ${ }^{14} \mathrm{~N}$ is mainly produced through ${ }^{11} \mathrm{~B}(\alpha, n){ }^{14} \mathrm{~N}$ ). On the contrary, in the outer region of the He-layer where $M_{r} \gtrsim 5.0 M_{\odot},{ }^{14} \mathrm{~N}$ is less abundant and the ${ }^{14} \mathrm{~N} /{ }^{4} \mathrm{He}$ ratio scatters widely owing to the adopted initial composition;

since the temperature during the explosion does not become high enough to proceed $\alpha$-capture reaction, ${ }^{14} \mathrm{~N}$ is produced through ${ }^{17} \mathrm{O}(n, \alpha){ }^{14} \mathrm{C}$ in place of ${ }^{11} \mathrm{~B}(\alpha, n){ }^{14} \mathrm{~N}$. In the H-rich envelope, ${ }^{14} \mathrm{~N}$ is produced through ${ }^{12} \mathrm{C}(p, \gamma){ }^{13} \mathrm{~N}\left(, e^{+} v_{\mathrm{e}}\right){ }^{13} \mathrm{C}$ $(p, \gamma)^{14} \mathrm{~N}$ and the range of the ${ }^{14} \mathrm{~N} /{ }^{4} \mathrm{He}$ ratio naturally becomes large owing to the difference of the ${ }^{12} \mathrm{C}$ content.

The distribution of the ${ }^{18} \mathrm{O} /{ }^{4} \mathrm{He}$ ratio, shown in Fig. 5(b), behaves very similarly to the case of the ${ }^{14} \mathrm{~N} /{ }^{4} \mathrm{He}$ ratio. In the inner region of the He-layer where $M_{r} \lesssim 4.7 M_{\odot},{ }^{18} \mathrm{O}$ is abundant and is scaled well by ${ }^{4} \mathrm{He}$. In the outer He-layer and in the $\mathrm{H}$-rich envelope, ${ }^{18} \mathrm{O}$ is produced from ${ }^{16} \mathrm{O}$ through two $n$-captures and two $p$-captures, respectively. As a result, ${ }^{18} \mathrm{O}$ is less abundant in such regions and the ${ }^{18} \mathrm{O} /{ }^{4} \mathrm{He}$ ratio varies over a wide range owing to the ${ }^{16} \mathrm{O}$-content.

4.2.5 ${ }^{6} \mathbf{L i},{ }^{7} \mathbf{L i}$, and ${ }^{9} \mathrm{Be} \quad$ Finally, we present distributions of the numbers of ${ }^{6} \mathrm{Li},{ }^{7} \mathrm{Li}$, and ${ }^{9} \mathrm{Be}$ normalized by the ${ }^{4} \mathrm{He}$ content in Fig. 6. In the He-layer, the ${ }^{7} \mathrm{Li} /{ }^{4} \mathrm{He}$ ratio is insensitive to the initial chemical compositions; the ratio is confined within a factor of 2 or so. As seen from Table 5, the production path of ${ }^{7} \mathrm{Li}$ is $\left.{ }^{3} \mathrm{He}(\alpha, \gamma){ }^{7} \mathrm{Be}(n, p)\right)^{7} \mathrm{Li}(\alpha, \gamma){ }^{11} \mathrm{~B}$ in the inner region of the He-layer. In most models, the amounts of ${ }^{4} \mathrm{He}$ and neutrons are almost identical, so that the final ${ }^{7} \mathrm{Li} /{ }^{4} \mathrm{He}$ ratio scarcely changes in these models. In models A3 and A4 with the abundant ${ }^{12} \mathrm{C}$ content, the number of neutrons decreases and ${ }^{7} \mathrm{Be}$ is left as it is. In the outer region of the He-layer where $M_{r} \gtrsim 5.4 M_{\odot},{ }^{7} \mathrm{Li} /{ }^{4} \mathrm{He}$ ratio is determined uniquely irrelevant to the adopted model. In this region, ${ }^{7} \mathrm{Li}$ is produced from ${ }^{4} \mathrm{He}$ and ${ }^{3} \mathrm{H}$ through 
${ }^{4} \mathrm{He}\left(v, v^{\prime} p\right)^{3} \mathrm{H}(\alpha, \gamma){ }^{7} \mathrm{Li}$ and is not decomposed. In the inner region of the H-rich envelope where $M_{r} \lesssim 7.6 M_{\odot}$, the variety of ${ }^{7} \mathrm{Li} /{ }^{4} \mathrm{He}$ is relatively small but becomes large in the outer region. In the outer region ${ }^{7} \mathrm{Li}$ is produced from ${ }^{12} \mathrm{C}$ mainly during the $v$-process stage. As a result, the ${ }^{7} \mathrm{Li} /{ }^{12} \mathrm{C}$ ratio is confined within a factor of 3 .

For ${ }^{6} \mathrm{Li}$ and ${ }^{9} \mathrm{Be}$, their ratios to ${ }^{4} \mathrm{He}$ scatters very widely in most of region but not in the middle region $\left(5.4 M_{\odot} \lesssim M_{r} \lesssim\right.$ $6.5 M_{\odot}$ ) where they are abundant (the ratios are confined within a factor of 6 or so). In the middle region, ${ }^{9} \mathrm{Be}$ is produced from ${ }^{7} \mathrm{Li}$ through ${ }^{7} \mathrm{Li}(t, n){ }^{9} \mathrm{Be}$ and ${ }^{6} \mathrm{Li}$ is produced through ${ }^{9} \mathrm{Be}(p, \alpha){ }^{6} \mathrm{Li}$. Or, more explicitly, they are produced from ${ }^{4} \mathrm{He}$ originally (see Table 5). In the other regions, ${ }^{9} \mathrm{Be}$ is produced from ${ }^{12} \mathrm{C}$, so that it is natural that both ${ }^{9} \mathrm{Be} /{ }^{4} \mathrm{He}$ and ${ }^{6} \mathrm{Li} /{ }^{4} \mathrm{He}$ ratios have large varieties due to the initial chemical composition. However, it is not so important because of their extremely small amounts.

\subsection{Decomposition of the light elements}

It is also interesting to see the decomposition process of the light elements which would exist in the presupernova stage. As mentioned in Section 3.2.2, we put $1 \times 10^{-6}$ and $1 \times 10^{-10}$ as the initial mass fractions of the rare $\mathrm{CNO}$-elements and the $X$-elements, respectively, keeping the amounts of the four major elements of model SII (such a model is called model B). The final mass fractions of the $X$-elements in model $\mathrm{B}$ are presented in Fig. 7(a). In the convective Helayer, the $X$-elements, which exist initially, are decomposed or hidden completely by those produced during the explosion. In fact, the distribution of the final mass fractions of the $X$-elements in the convective He-layer is almost same as those in model SII (compare with Fig. 1(a)). In the radiative He-layer, a small part of initially existing ${ }^{6} \mathrm{Li}$ and ${ }^{9} \mathrm{Be}$ can survive against $(p, \alpha)$-reactions. In the $\mathrm{H}$-rich envelope, only ${ }^{7} \mathrm{Be}$ and ${ }^{11} \mathrm{C}$, both of which are $p$-rich unstable elements, are newly produced and remain against $p$-capture process. However, all of the stable $X$-elements $\left(\right.$ except $\left.{ }^{11} \mathrm{~B}\right)$ are not formed through the $v$-process but decomposed effectively owing to the shock heating. For ${ }^{11} \mathrm{~B}$, we can see from Fig. 7(a) that a

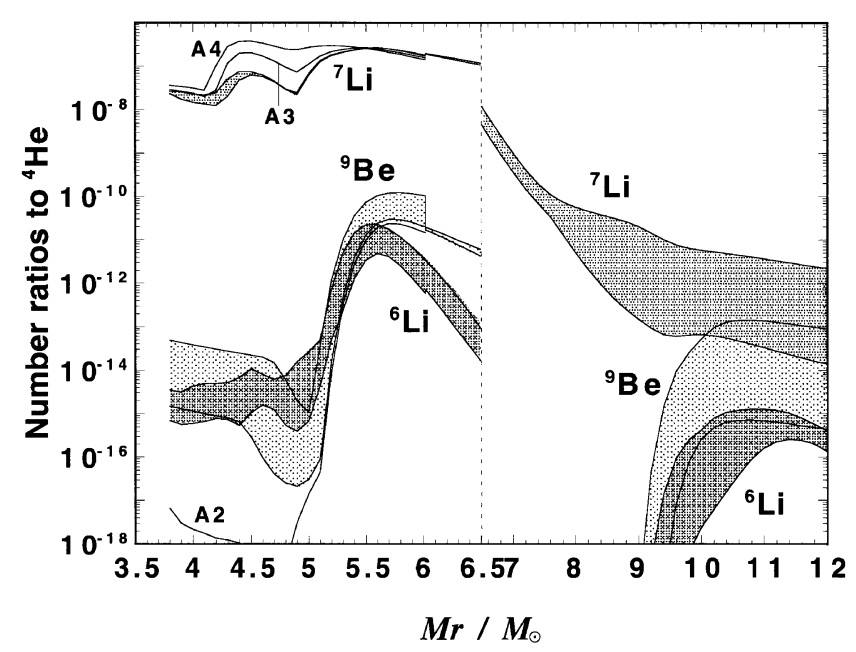

Fig. 6. Distributions of the number ratios of ${ }^{6} \mathrm{Li},{ }^{7} \mathrm{Li}$, and ${ }^{9} \mathrm{Be}$ to ${ }^{4} \mathrm{He}$. Dark shaded regions, medial shaded regions, and light shaded regions show ${ }^{6} \mathrm{Li} /{ }^{4} \mathrm{He},{ }^{7} \mathrm{Li} /{ }^{4} \mathrm{He}$, and ${ }^{9} \mathrm{Be} /{ }^{4} \mathrm{He}$ ratios in most models, respectively. A curve labeled $\mathrm{A} 2$ is of ${ }^{9} \mathrm{Be} /{ }^{4} \mathrm{He}$ ratio.
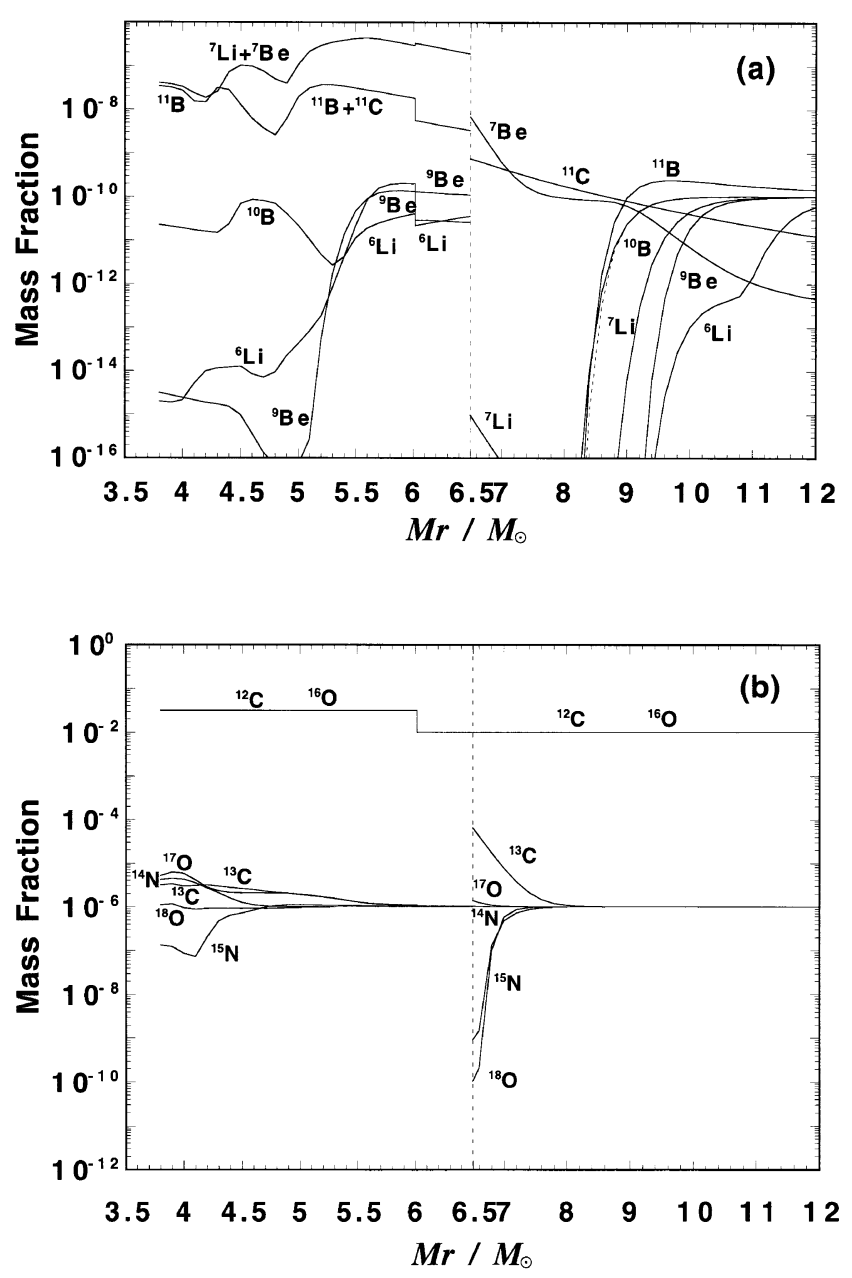

Fig. 7. Distributions of the final abundances of the $X$-elements (panel(a)) and the CNO-elements (panel (b)) in model B. In panel(a) dashed line denotes the fraction of survival ${ }^{11} \mathrm{~B}$ of the shock heating.

small amount of ${ }^{11} \mathrm{~B}$ is produced through the $\nu$-process. In other words, for the $X$-elements (except ${ }^{11} \mathrm{~B}$ ) the distribution curves in the H-rich envelope mean the fractions of survival of the shock heating; in the inner region they are completely decomposed and in the outer region $\left(M_{r} \gtrsim 12 M_{\odot}\right)$ they can survive and keep their initial compositions in the presupernova stage. For the later convenience, we also show the survival fraction of ${ }^{11} \mathrm{~B}$ by dashed line in Fig. 7(a), which is obtained from a numerical calculation without the $v$-process.

The distributions of the final mass fractions of the CNOelements are shown in Fig. 7(b). In the innermost region of the convective He-layer, only ${ }^{15} \mathrm{~N}$ is decomposed through ${ }^{15} \mathrm{~N}(p, \alpha){ }^{12} \mathrm{C}$. The other isotopes are produced and reach the abundance level in model SII. In the outer region where $M_{r} \gtrsim 4.5 M_{\odot}$ (including the radiative He-layer), all of the $\mathrm{CNO}$-elements conserve their initial abundances. In the $\mathrm{H}-$ rich envelope, ${ }^{15} \mathrm{~N}$ and ${ }^{18} \mathrm{O}$ are decomposed in the narrow region inner than 7.2 $M_{\odot}$. When the CNO-cycle is in quasisteady state, both ${ }^{15} \mathrm{~N} /{ }^{14} \mathrm{~N}$ and ${ }^{18} \mathrm{O} /{ }^{16} \mathrm{O}$ ratios are quite small, since ${ }^{15} \mathrm{~N}(p, \alpha){ }^{12} \mathrm{C}$ and ${ }^{18} \mathrm{O}(p, \alpha){ }^{15} \mathrm{~N}$ have relatively large cross sections compared with the other reactions of the CNOcycle. So it is natural that only ${ }^{15} \mathrm{~N}$ and ${ }^{18} \mathrm{O}$ are decomposed during the explosion. In the region outer than $M_{r} \gtrsim 7.8 M_{\odot}$, the CNO-elements keep completely their initial abundances. 

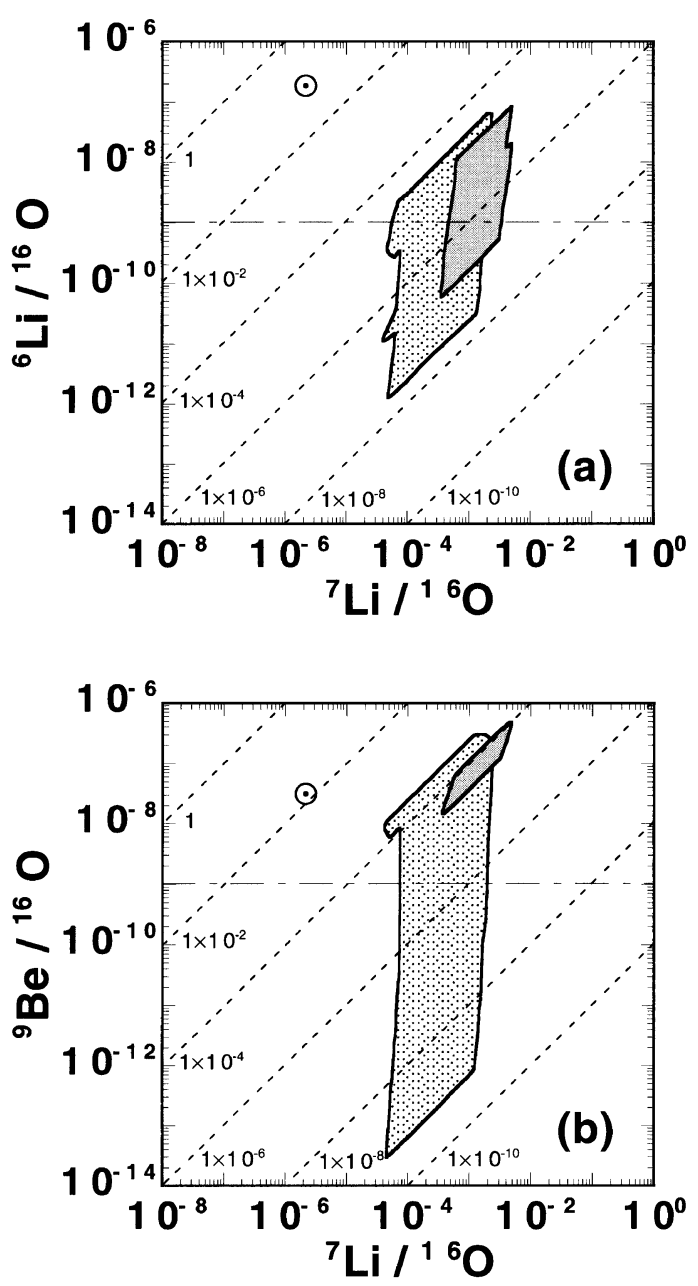

Fig. 8. Diagrams between ${ }^{6} \mathrm{Li} /{ }^{16} \mathrm{O}$ and ${ }^{7} \mathrm{Li} /{ }^{16} \mathrm{O}$ ratios (panel (a)) and between ${ }^{9} \mathrm{Be} /{ }^{16} \mathrm{O}$ and ${ }^{7} \mathrm{Li} /{ }^{16} \mathrm{O}$ ratios (panel (b)). The ratios are all in number. Light and dark shaded regions show the varieties of the ratios in the convective He-layer outer than $5.1 M_{\odot}$ and in the radiative He-layer, respectively. A dash-dotted horizontal line indicates the detection limit (see text). Furthermore, dotted lines show the values of constant ${ }^{6} \mathrm{Li} /{ }^{7} \mathrm{Li}$ ratio (a) and ${ }^{9} \mathrm{Be} /{ }^{7} \mathrm{Li}$ ratio (b). A mark $\odot$ denotes the ratios of the solar-system composition.

\section{Probable Ranges of the Isotopic/Elemental Ra- tios}

Combining the probable initial composition evaluated in Section 2 and the numerical results found in the last section, we will consider the probable ranges of the isotopic/elemental ratios, ${ }^{6} \mathrm{Li} /{ }^{7} \mathrm{Li},{ }^{9} \mathrm{Be} /{ }^{7} \mathrm{Li},{ }^{11} \mathrm{~B} /{ }^{7} \mathrm{Li},{ }^{12} \mathrm{C} /{ }^{13} \mathrm{C},{ }^{14} \mathrm{~N} /{ }^{15} \mathrm{~N}$, and ${ }^{16} \mathrm{O} /$ ${ }^{17} \mathrm{O}$, which are to be observed in supernova ejecta.

\subsection{The ${ }^{6} \mathrm{Li} /{ }^{7} \mathrm{Li}$ and ${ }^{9} \mathrm{Be} /{ }^{7} \mathrm{Li}$ ratios}

In Paper I we showed that only small amounts of ${ }^{6} \mathrm{Li}$ and ${ }^{9} \mathrm{Be}$ are produced in the He-layer and the $\mathrm{H}$-rich envelope during the supernova explosion and that the ratios of them to ${ }^{7} \mathrm{Li}$ are very small compared with, say, the solar ratios. These are the remarkable features of nucleosynthesis in the supernova explosion and, hence, it is important to confirm whether these arguments are proper or not even when we choose the different initial chemical compositions from Paper I.

As conjectured from Fig. 6, the produced amounts of ${ }^{6} \mathrm{Li}$ and ${ }^{9} \mathrm{Be}$ become large compared with the detection limit (which is an abundance level of $1 \times 10^{-9}$ times as small as the number of ${ }^{16} \mathrm{O}$; see Paper I) only in the outer region of the convective He-layer. This is always true irrelevant to the initial contents of ${ }^{6} \mathrm{Li}$ and ${ }^{9} \mathrm{Be}$ because, as mentioned in Section 4.3, pre-existing ${ }^{6} \mathrm{Li}$ and ${ }^{9} \mathrm{Be}$ are almost completely decomposed in the He-layer and the inner H-rich envelope. In Figs. 8(a) and 8(b), we show the probable ranges of the final ${ }^{6} \mathrm{Li}$ and ${ }^{9} \mathrm{Be}$ contents in ${ }^{6} \mathrm{Li}$ and ${ }^{9} \mathrm{Be}$ abundant region in the convective He-layer $\left(M_{r} \geq 5.1 M_{\odot}\right)$ and in the radiative He-layer. In these diagrams, the results of models A2 and A4 in the convective He-layer and model A1 in the radiative He-layer are excluded because of a mismatch in the ${ }^{12} \mathrm{C}$ and ${ }^{16} \mathrm{O}$ contents. Furthermore, the amount of ${ }^{16} \mathrm{O}$ of each model is replaced by that within the probable range. Figures show that the ${ }^{6} \mathrm{Li} /{ }^{7} \mathrm{Li}$ and the ${ }^{9} \mathrm{Be} /{ }^{7} \mathrm{Li}$ ratios are always less than $3 \times 10^{-5}$ and $2 \times 10^{-4}$, respectively. Thus, we can say that ${ }^{6} \mathrm{Li}$ and ${ }^{9} \mathrm{Be}$ are not produced so much during the supernova explosion in the He-layer and in the H-rich envelope and that their ratios to ${ }^{7} \mathrm{Li}$ are much smaller than those of the solarsystem composition. It should be noted, however, that it is possible to conserve their initial ratios (of the levels given by Eqs. (2) and (3)) in the outermost part of the H-rich envelope where $M_{r} \gtrsim 10 M_{\odot}$ (see Fig. 7(a)).

\subsection{The ${ }^{11} \mathrm{~B} /{ }^{7} \mathrm{Li}$ ratio}

As seen in Section 4.2, the amounts of ${ }^{11} \mathrm{~B}$ and ${ }^{7} \mathrm{Li}$ are scaled conveniently by those of different major constituents (i.e., ${ }^{12} \mathrm{C}$ and ${ }^{4} \mathrm{He}$, respectively) and their final abundances suffer from their initial contents in the H-rich envelope (see Section 4.3), which we do not know precisely. Moreover, since the amount of ${ }^{14} \mathrm{~N}$ is relatively large in the H-rich envelope (see Section 2.1), we should take account of ${ }^{7} \mathrm{Li}$ production through ${ }^{14} \mathrm{~N}\left(\nu, v^{\prime} \alpha\right){ }^{10} \mathrm{~B}(p, \alpha){ }^{7} \mathrm{Be}\left(e^{-}, v_{\mathrm{e}}\right){ }^{7} \mathrm{Li}$ which may play an important role on the ${ }^{7} \mathrm{Li}$ production in the intermediate mass region (see Paper I). Thus, it is not so easy to find the probable ratio of the ${ }^{11} \mathrm{~B} /{ }^{7} \mathrm{Li}$. Here, by the help of Paper I as well as our numerical simulations mentioned in Section 4 , we evaluate the probable range of the ${ }^{11} \mathrm{~B} /{ }^{7} \mathrm{Li}$ ratio by the following way. The result is illustrated in Fig. 9(a).

In the convective He-layer, pre-existing ${ }^{7} \mathrm{Li}$ and ${ }^{11} \mathrm{~B}$ are completely decomposed on the way to the presupernova stage and the ${ }^{11} \mathrm{~B} /{ }^{7} \mathrm{Li}$ ratio is determined by the nuclear processes during the supernova explosion. In the inner region of the He-layer the probable range of the ${ }^{11} \mathrm{~B} / 7 \mathrm{Li}$ ratio is evaluated almost uniquely from our results of models SII, A1, and $\mathrm{A} 3$, of which ${ }^{12} \mathrm{C}$ and ${ }^{16} \mathrm{O}$-contents are consistent with the probable initial abundances shown in Table 3. In the outer convective He-layer (where $M_{r} \gtrsim 5.2 M_{\odot}$ ) as well as in the radiative He-layer, ${ }^{7} \mathrm{Li}$ and ${ }^{11} \mathrm{~B}$ are produced mainly from ${ }^{4} \mathrm{He}$ and ${ }^{12} \mathrm{C}$, respectively. Hence, the range of the ${ }^{11} \mathrm{~B} /{ }^{7} \mathrm{Li}$ ratio is governed essentially by the ${ }^{12} \mathrm{C} /{ }^{4} \mathrm{He}$ ratio and evaluated from

$$
\frac{{ }^{11} \mathrm{~B}}{{ }^{7} \mathrm{Li}}=\left(\frac{{ }^{12} \mathrm{C}}{{ }^{4} \mathrm{He}}\right)_{\mathrm{o}}\left(\frac{{ }^{11} \mathrm{~B}}{{ }^{12} \mathrm{C}}\right)_{\mathrm{m}}\left(\frac{{ }^{7} \mathrm{Li}}{{ }^{4} \mathrm{He}}\right)_{\mathrm{m}}^{-1} .
$$

In the above, $\left({ }^{12} \mathrm{C} /{ }^{4} \mathrm{He}\right)_{0}$ is the initial elemental ratio given in Tables 2 and 3. The ratios $\left({ }^{11} \mathrm{~B} /{ }^{12} \mathrm{C}\right)_{\mathrm{m}}$ and $\left({ }^{7} \mathrm{Li} /{ }^{4} \mathrm{He}\right)_{\mathrm{m}}$ are found from the results of our calculations mentioned in Section 4 (i.e., the shaded bands in Figs. 3(b) and 6).

In the inner H-rich envelope where $M_{r} \lesssim 8.5 M_{\odot}$, both ${ }^{7} \mathrm{Li}$ and ${ }^{11} \mathrm{~B}$ existing in the presupernova stage are decomposed almost completely by the shock heating and re-produced 

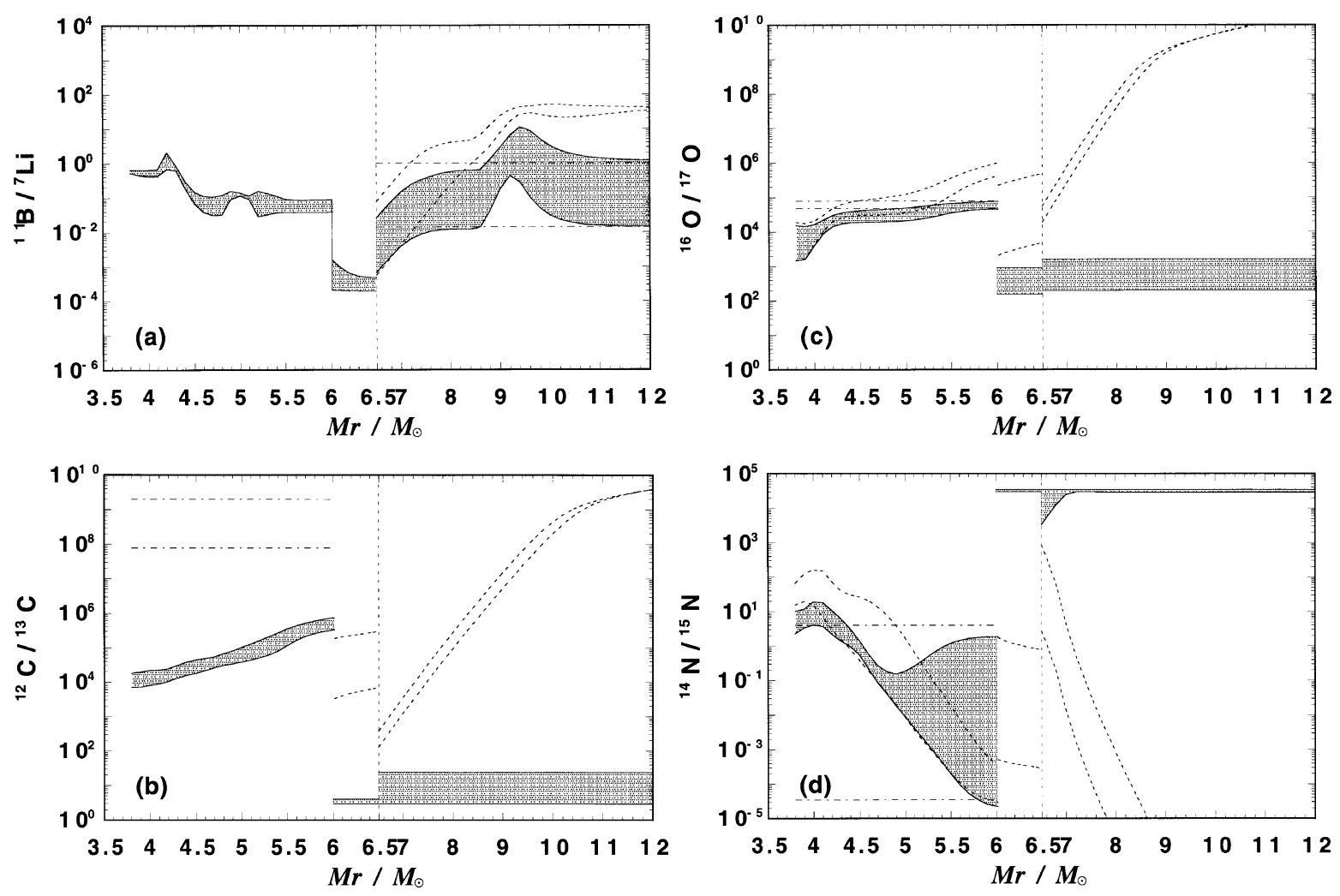

Fig. 9. Distributions of the number ratios of ${ }^{11} \mathrm{~B} /{ }^{7} \mathrm{Li}(\operatorname{panel}(\mathrm{a})),{ }^{12} \mathrm{C} /{ }^{13} \mathrm{C}$ (panel(b)), ${ }^{14} \mathrm{~N} /{ }^{15} \mathrm{~N}(\operatorname{panel}(\mathrm{c}))$, and ${ }^{16} \mathrm{O} /{ }^{17} \mathrm{O}(\mathrm{panel}(\mathrm{d}))$. Shaded regions show the probable ratios evaluated from the results of numerical simulations and the probable chemical compositions in the presupernova stage. Regions between two dashed lines correspond to the shaded regions in Figs. 2 to 5, i.e., the probable ranges of the ratios of the produced elements and regions between two dash-dotted lines present the probable ranges of the elemental ratios in the presupernova stage evaluated in Section 2.

through the $v$-processes and the explosive nucleosynthesis (see Section 4.3). In the outer region of the H-rich envelope $\left(M_{r} \gtrsim 10 M_{\odot}\right)$, both ${ }^{7} \mathrm{Li}$ and ${ }^{11} \mathrm{~B}$ are formed commonly through the $v$-process from ${ }^{12} \mathrm{C}$ during the supernova explosion but their produced amounts are very small, as seen in Fig. 1(a), compared with those of ${ }^{7} \mathrm{Li}$ and ${ }^{11} \mathrm{~B}$ observed in stellar surfaces (see Section 2.2). Thus, in such regions of the H-rich envelope, the ${ }^{11} \mathrm{~B} /{ }^{7} \mathrm{Li}$ ratio is described by

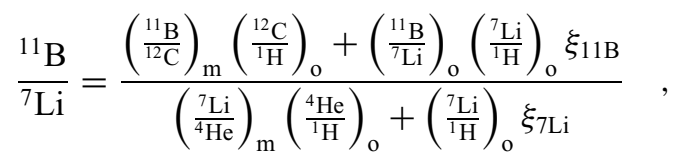

where subscripts $m$ and o denote the elemental ratios found in our model calculations and the probable ratios predicted in Section 4, respectively. Furthermore, $\xi_{i}$ denotes the fraction of survival of the shock heating to the initial amount of $i$-th constituent and is shown in Fig. 7(a).

In the intermediate region where $8.5 M_{\odot} \lesssim M_{r} \lesssim 10 M_{\odot}$, as mentioned at the top of this subsection, we should add the amount of ${ }^{7} \mathrm{Li}$ produced from ${ }^{14} \mathrm{~N}$ to denominator of Eq. (8), i.e., $\left({ }^{7} \mathrm{Be} /{ }^{14} \mathrm{~N}\right)_{\mathrm{m}} \cdot\left({ }^{14} \mathrm{~N} /{ }^{1} \mathrm{H}\right)_{\mathrm{o}}$. Here the ratio of $\left({ }^{7} \mathrm{Be} /{ }^{14} \mathrm{~N}\right)_{\mathrm{m}}$ is evaluated from the numerical result in Paper $\mathrm{I}^{*}$ and the adopted range of the ratio is between $2 \times 10^{-10}$ and $8 \times 10^{-9}$ in the corresponding mass range. The probable range of the amount of ${ }^{14} \mathrm{~N}$, that is, $\left({ }^{14} \mathrm{~N} /{ }^{1} \mathrm{H}\right)_{\mathrm{o}}$, is written in Section 2.1 .

The ratio of $\left({ }^{7} \mathrm{Be} /{ }^{14} \mathrm{~N}\right)_{\mathrm{m}}$ is evaluated as the difference between the ${ }^{10} \mathrm{~B} /{ }^{14} \mathrm{~N}$ ratio produced through ${ }^{14} \mathrm{~N}\left(v, v^{\prime} \alpha\right){ }^{10} \mathrm{~B}$, which is not apparently written in Paper I, and the final ${ }^{10} \mathrm{~B} /{ }^{14} \mathrm{~N}$ ratio.

\subsection{The ${ }^{12} \mathrm{C} /{ }^{13} \mathrm{C}$ and ${ }^{16} \mathrm{O} /{ }^{17} \mathrm{O}$ ratios}

We can readily find the probable ${ }^{12} \mathrm{C} /{ }^{13} \mathrm{C}$ ratio as long as the initial chemical compositions are included in the probable ranges listed in Tables 1 to 3 . In the H-rich envelope and the radiative He-layer, the probable content of ${ }^{13} \mathrm{C}$ is much larger than that produced during the supernova explosion (see Fig. 2(b)) and, as seen in Section $4.3,{ }^{13} \mathrm{C}$ is hardly decomposed. Thus, in these regions the ${ }^{12} \mathrm{C} /{ }^{13} \mathrm{C}$ ratio is entirely determined by the initial ratio. On the other hand, in the convective He-layer, the amount of ${ }^{13} \mathrm{C}$ expected in the presupernova stage is of the order of $1 \times 10^{-10}$, which is much less than that produced during the supernova explosion (see Table 3 and Fig. 2(b)); in this region, the ${ }^{12} \mathrm{C} /{ }^{13} \mathrm{C}$ ratio is a result of the nuclear processes during the explosion and the distribution is well described by the shaded band illustrated in Fig. 2(b). Here we exclude models A2 and A4 because of an extreme mismatch in the initial contents of ${ }^{12} \mathrm{C}$ and ${ }^{16} \mathrm{O}$ (compare Table 3 with Table 4). The result is shown in Fig. 9(b).

Evaluation of the probable ${ }^{16} \mathrm{O} /{ }^{17} \mathrm{O}$ ratio is slightly complicated compared with the case of ${ }^{12} \mathrm{C} /{ }^{13} \mathrm{C}$. In the radiative He-layer and the $\mathrm{H}$-rich envelope, the amount of ${ }^{17} \mathrm{O}$ in the presupernova stage is much larger than that produced in the supernova explosion similarly to the case of ${ }^{13} \mathrm{C}$. Furthermore, ${ }^{17} \mathrm{O}$ is hardly decomposed, as seen in Section 4.3, in these regions and thus, the ${ }^{16} \mathrm{O} /{ }^{17} \mathrm{O}$ ratio is simply determined by that of the presupernova stage. However, the amount of ${ }^{17} \mathrm{O}$ produced in the supernova explosion is slightly larger 
than that expected in the presupernova stage in the inner region but not in the outer region of the convective He-layer. Thus, the distribution of the ${ }^{16} \mathrm{O} /{ }^{17} \mathrm{O}$ ratio is given, as shown in Fig. 9(c), by the inverse of the sum of the ${ }^{17} \mathrm{O} /{ }^{16} \mathrm{O}$ ratio shown by the shaded band of Fig. 4(b) and that of the probable initial ratio shown in Table 3 . The ${ }^{16} \mathrm{O} /{ }^{17} \mathrm{O}$ ratio is determined by the explosive nucleosynthesis in the inner region $\left(M \lesssim 5 M_{\odot}\right)$ and by the initial ratio in the outer region. 5.4 The ${ }^{14} \mathrm{~N} /{ }^{15} \mathrm{~N}$ ratio

In evaluating the probable range of the ${ }^{14} \mathrm{~N} /{ }^{15} \mathrm{~N}$ ratio, we have to pay special attention to the following four. The first is that, in our present study, ${ }^{14} \mathrm{~N}$ is not included as an initial constituent although ${ }^{14} \mathrm{~N}$ is the most abundant isotope among the CNO-elements in the radiative He-layer and the $\mathrm{H}$-rich envelope. Secondly, ${ }^{18} \mathrm{O}$ is not included too though ${ }^{15} \mathrm{~N}$ is possibly formed from ${ }^{18} \mathrm{O}$, at least, in the innermost convective He-layer (see Paper I). Thirdly, it is quite uncertain how much amount of ${ }^{14} \mathrm{~N}$ is contained in the convective He-layer (see Table 3) in the presupernova stage. Finally, the two isotopes are formed from different parent isotopes: ${ }^{15} \mathrm{~N}$ is mainly produced through ${ }^{16} \mathrm{O}\left(v, v^{\prime} p\right){ }^{15} \mathrm{~N}$ and ${ }^{14} \mathrm{~N}$ through ${ }^{11} \mathrm{~B}(\alpha, n){ }^{14} \mathrm{~N}$. Therefore, the ${ }^{14} \mathrm{~N} /{ }^{15} \mathrm{~N}$ ratio depends on the contents of ${ }^{4} \mathrm{He}$ and ${ }^{16} \mathrm{O}$ in a complicated manner.

Now, we will evaluate the probable range of the ${ }^{14} \mathrm{~N} /{ }^{15} \mathrm{~N}$ ratio in the convective He-layer. Since ${ }^{14} \mathrm{~N}$ is not decomposed during the explosion, the final abundance is given by the sum of the abundance in the presupernova stage and that produced during the explosion. For ${ }^{15} \mathrm{~N}$, we first note that, as seen in Table 3 , the content of ${ }^{14} \mathrm{~N}$ is very small in the convective He-layer and, hence, the produced amount of ${ }^{15} \mathrm{~N}$ is not so affected by the initial content of ${ }^{14} \mathrm{~N}$. In the innermost region, ${ }^{15} \mathrm{~N}$ is decomposed to the level of $10 \%$ of the initial abundance (see Fig. 7(b)). However, it is re-produced probably through ${ }^{18} \mathrm{O}(p, \alpha){ }^{15} \mathrm{~N}$ to the abundance level more than the initial composition (see Paper I). The produced amount of ${ }^{15} \mathrm{~N}$ from ${ }^{16} \mathrm{O}$ is readily found from our present study. The amount of ${ }^{15} \mathrm{~N}$ from ${ }^{18} \mathrm{O}$ is also estimated from the result of Paper I in which we found that the final ratio of ${ }^{15} \mathrm{~N} /{ }^{18} \mathrm{O}$ is $3 \times$ $10^{-3}$ to $3 \times 10^{-1}$ (for the probable values of the ${ }^{18} \mathrm{O} /{ }^{16} \mathrm{O}$ ratio, see Table 3). Thus, we find the amount of ${ }^{15} \mathrm{~N}$ as the sum of the initial abundance and that produced by the two ways during the explosion. The probable range of the ${ }^{14} \mathrm{~N} /{ }^{15} \mathrm{~N}$ ratio in the convective He-layer, evaluated by the way mentioned above (note that we exclude the results of models $\mathrm{A} 2$ and $\mathrm{A} 4$ because of a mismatch in the ${ }^{12} \mathrm{C}$ and ${ }^{16} \mathrm{O}$-contents), is shown in Fig. 9(d). In the inner region where $M_{r} \lesssim 5.0 M_{\odot}$, the uncertainty in the ${ }^{14} \mathrm{~N} /{ }^{15} \mathrm{~N}$ ratio attributes mainly to that of the ${ }^{16} \mathrm{O} /{ }^{4} \mathrm{He}$ ratio. In the outer region, the maximum value of the ratio is determined by the initial ratio and the minimum one is determined by the ratio obtained from our present results. The range of the probable ratio becomes larger with an increase in the mass coordinate because of the large uncertainty in the abundance of pre-existing ${ }^{14} \mathrm{~N}$.

In the radiative He-layer and most region of the H-rich envelope, the contents of ${ }^{14} \mathrm{~N}$ and ${ }^{15} \mathrm{~N}$ in the presupernova stage are much larger than those produced during the explosion. Furthermore, ${ }^{15} \mathrm{~N}$ production from ${ }^{14} \mathrm{~N}$ can be excluded as mentioned before. As a result, the probable range of the ${ }^{14} \mathrm{~N} /{ }^{15} \mathrm{~N}$ ratio is entirely governed by the isotopic ra- tio in the presupernova stage. However, in the innermost region of the $\mathrm{H}$-rich envelope the ${ }^{14} \mathrm{~N} /{ }^{15} \mathrm{~N}$ ratio differs from the initial values. According to the result of model B mentioned in Section 4.3, pre-existing ${ }^{15} \mathrm{~N}$ is decomposed during the explosion in such a region and, at the same time, ${ }^{15} \mathrm{~N}$ would be produced from ${ }^{14} \mathrm{~N}$ by the shock heating through ${ }^{14} \mathrm{~N}(p, \gamma){ }^{15} \mathrm{O}\left(, e^{+} v_{\mathrm{e}}\right){ }^{15} \mathrm{~N}$, if there exists a sufficient amount of ${ }^{14} \mathrm{~N}$. In fact, Paper I tells us this is true in the present case since the initial ${ }^{14} \mathrm{~N} /{ }^{15} \mathrm{~N}$ ratio is as large as $3 \times 10^{4}$ (see Table 1). Thus, in the innermost region of the H-rich envelope the ${ }^{14} \mathrm{~N} /{ }^{15} \mathrm{~N}$ ratio is governed by the explosive nuclear processes; according to Paper I, the ratio is in the range between $3 \times 10^{3}$ and $3 \times 10^{4}$.

\section{Summary and Conclusions}

In a series of our studies, we have investigated the elemental/isotopic ratios of the light elements synthesized during the supernova explosion in order to find suitable criteria for distinguishing presolar grains of supernova origin from those of other origins. In this study, we first deduced probable ranges of the amounts and the isotopic ratios of the light elements in the presupernova stage on the basis of the previous studies on stellar evolution and atmospheric compositions of stars. Next, we investigated the influence of the initial chemical composition on the light element synthesis by the use of artificially modeled compositions which consist of only ${ }^{1} \mathrm{H},{ }^{4} \mathrm{He}$, ${ }^{12} \mathrm{C}$, and ${ }^{16} \mathrm{O}$. We also made a simulation of decomposition processes of the light elements during the supernova explosion using a model with over-abundant rare light elements. The main results of our numerical simulations (described in Section 4) are summarized as follows:

1) Roughly speaking, the light elements produced in the supernova explosion are classified into three groups, i.e., (a) $)^{6} \mathrm{Li},{ }^{7} \mathrm{Li},{ }^{9} \mathrm{Be},{ }^{14} \mathrm{~N}$, and ${ }^{18} \mathrm{O},(\mathrm{b}){ }^{10} \mathrm{~B},{ }^{11} \mathrm{~B}$, and ${ }^{13} \mathrm{C}$, and $(\mathrm{c}){ }^{15} \mathrm{~N}$ and ${ }^{17} \mathrm{O}$. The final productions of elements of groups (a), (b), and (c) can be properly scaled by the amounts of ${ }^{4} \mathrm{He},{ }^{12} \mathrm{C}$, and ${ }^{16} \mathrm{O}$ contained in the presupernova stage, respectively.

2) In the radiative He-layer and the H-rich envelope, the final abundances of the CNO-elements are governed by the chemical composition in the presupernova stage in a sense that pre-existing elements survive as they were.

Combining the results presented in Sections 2 and 4, we constructed the probable distributions of the final elemental/isotopic ratios of the light elements of ${ }^{11} \mathrm{~B} /{ }^{7} \mathrm{Li},{ }^{12} \mathrm{C} /{ }^{13} \mathrm{C}$, ${ }^{16} \mathrm{O} /{ }^{17} \mathrm{O}$, and ${ }^{14} \mathrm{~N} /{ }^{15} \mathrm{~N}$. We also confirmed the result of $\mathrm{Pa}-$ per I that the amounts of ${ }^{6} \mathrm{Li}$ and ${ }^{9} \mathrm{Be}$ produced in the He-layer and the inner region of the $\mathrm{H}$-rich envelope are much smaller than that of ${ }^{7} \mathrm{Li}$ and that in the outermost region of the $\mathrm{H}$-rich envelope the initial ratios are conserved.

Now, according to Paper I, we make three diagrams between two isotopic/elemental ratios which are useful for the study of presolar grains of supernova origin: diagrams of ${ }^{11} \mathrm{~B} /{ }^{7} \mathrm{Li}^{12} \mathrm{C} /{ }^{13} \mathrm{C},{ }^{14} \mathrm{~N} /{ }^{15} \mathrm{~N}-{ }^{12} \mathrm{C} /{ }^{13} \mathrm{C}$, and ${ }^{16} \mathrm{O} /{ }^{17} \mathrm{O}-{ }^{12} \mathrm{C} /{ }^{13} \mathrm{C}$. They are illustrated in Figs. 10(a) to $10(\mathrm{c})$. In these figures, four kinds of regions are overwritten: hatched regions, dark shadow regions, light shadow regions, and regions enclosed by dashed curves. A combined set of the dark shadow region 

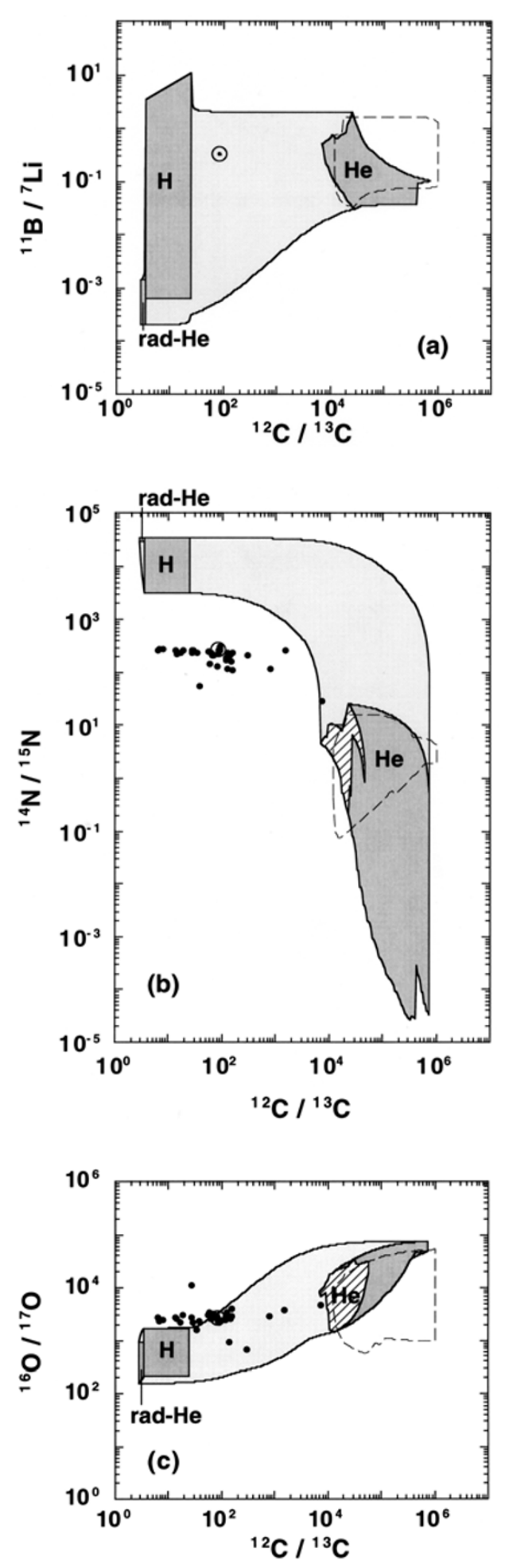

Fig. 10. The number ratios of ${ }^{11} \mathrm{~B} /{ }^{7} \mathrm{Li}(\operatorname{panel}(\mathrm{a})),{ }^{14} \mathrm{~N} /{ }^{15} \mathrm{~N}$ (panel(b)), and ${ }^{16} \mathrm{O} /{ }^{17} \mathrm{O}$ (panel(c)) versus ${ }^{12} \mathrm{C} /{ }^{13} \mathrm{C}$. Three regions with a dark shade are of the convective He-layer (denoted by $\mathrm{He}$ ), of the radiative He-layer (denoted by rad-He), and of the H-rich envelope (denoted by $\mathrm{H}$ ) whereas a region with a light shade denotes the spread of the ratios due to mixing between the two regions. The hatched regions denote the ratios which are peculiar to the nucleosynthesis in the supernova explosion determined independently of the chemical composition in the presupernova stage. Furthermore, the region enclosed by dashed curves corresponds to the dark shaded region of the convective He-layer shown in Paper I. In panels (b) and (c) small closed circles show the isotopic ratios of low density graphite grains, of which ${ }^{12} \mathrm{C} /{ }^{13} \mathrm{C},{ }^{14} \mathrm{~N} /{ }^{15} \mathrm{~N}$, and ${ }^{16} \mathrm{O} /{ }^{17} \mathrm{O}$ ratios were measured. The adopted isotopic ratios are listed in table 3 of Travaglio et al. (1999). and the hatched region, constructed from the shaded regions of Fig. 9, show the probable ranges of the isotopic/elemental ratios. The probable ratios are confined within relatively small two regions in each diagram; one is of the H-rich envelope (including the radiative He-layer) and the other is of the convective He-layer. The hatched regions mean that the ratios are completely determined by the nuclear processes during the supernova explosion irrelevant to the chemical compositions in the presupernova stage. In other words, the isotopic/elemental ratios belonging to the hatched regions are of peculiar to the nucleosynthesis during the supernova explosion. Furthermore, the light shadow regions denote the dispersion of the ratios due to a material mixing between the He-layer and the H-rich envelope, which would be triggered by the Rayleigh-Taylor instability during the explosion (e.g., Hachisu et al., 1994). The regions enclosed by dashed curves correspond to the dark shadow regions found in Paper I, in which we investigated the isotopic/elemental ratios under a variety of the neutrino emission models.

The three isotopic/elemental diagrams shown in Fig. 10 are nearly identical to those found in Paper I. In the convective He-layer, the regions of the isotopic/elemental ratios are almost included in the regions of Paper I, which are enclosed by dashed curves in Fig. 10, except the ${ }^{14} \mathrm{~N} /{ }^{15} \mathrm{~N}$ ratio. In the radiative He-layer, the regions of the isotopic/elemental ratios in Fig. 10 are almost similar to those in the corresponding figures in Paper I. In the H-rich envelope, the regions of the ratios are mainly determined by the probable ranges of the ratios in the presupernova stage. So, the regions of the ratios obtained in this study are larger than those obtained in Paper I (in which we did not take into account a variety of the initial chemical compositions). The regions of the ratios due to the material mixing are essentially similar to those obtained from Paper I.

We should note the ${ }^{14} \mathrm{~N} /{ }^{15} \mathrm{~N}$ ratio which shows a different feature between Fig. 10 and Fig. 14 in Paper I; in the outer region of the convective He-layer (where $M_{r} \gtrsim 5 M_{\odot}$ ), the ${ }^{14} \mathrm{~N} /{ }^{15} \mathrm{~N}$ ratio becomes very small, as seen in Fig. $10(\mathrm{~b})$, compared with the results in Paper I where the minimum value of the ${ }^{14} \mathrm{~N} /{ }^{15} \mathrm{~N}$ ratio is about $3 \times 10^{-2}$ (see the dashed curve in Fig. 10(b)). This is due to the large uncertainty of the amount of ${ }^{14} \mathrm{~N}$ in the presupernova stage (see Section 5.4). However, the region of the ${ }^{14} \mathrm{~N} /{ }^{15} \mathrm{~N}$ ratio below $1 \times 10^{-2}$ is not so important on the study of the presolar grains of supernova origin because the abundance of ${ }^{14} \mathrm{~N}$ is extremely small compared with that of ${ }^{16} \mathrm{O}$.

Thus, we arrive at the following conclusions.

1) A variety of the chemical compositions in the presupernova stage does not affect so much the isotopic/elemental ratios (which we perceive) compared with a variety of the neutrino emission models investigated in Paper I as long as we adopt the initial chemical composition within the probable range mentioned in Section 2.

2) The hatched regions in Fig. 10, in which the ratios are determined by the nuclear processes during the supernova explosion irrelevant to the adopted chemical composition in the presupernova stage, are almost included in the regions brought about by the uncertainty in the neutrino emission model in Paper I. 
3) In the H-rich envelope, the regions of the ratios are almost determined by the chemical composition in the presupernova stage, and thus, the results of Paper I should be replaced by those of the present study.

4) As in the case of Paper I, we propose that presolar grains with ${ }^{6} \mathrm{Li} /{ }^{7} \mathrm{Li}$ less than $3 \times 10^{-5}$ and with ${ }^{9} \mathrm{Be} /{ }^{7} \mathrm{Li}$ less than $2 \times 10^{-4}$ should be originated from the supernova, even if we consider an uncertainty in the chemical composition in the presupernova stage.

In short, we can say that the conclusions found in Paper I (see Introduction) are proper without any significant modification even if we take into account of the uncertainty in the chemical composition in the presupernova stage. Furthermore, as pointed out in Paper I, the isotopic diagrams shown in Figs. 8 and 10 are useful for studying the presolar grains of supernova origin.

We have a brief comment on the comparison of Fig. 10 with isotopic ratios of 35 low density graphite grains, for which ${ }^{12} \mathrm{C} /{ }^{13} \mathrm{C},{ }^{14} \mathrm{~N} /{ }^{15} \mathrm{~N}$, and ${ }^{16} \mathrm{O} /{ }^{17} \mathrm{O}$ ratios were listed in Travaglio et al. (1999). The isotopic ratios summarized in Travaglio et al. (1999) were originally reported by Amari et al. (1995), (1996a), (1996b), Nittler et al. (1996), and some of them had been unpublished. The ratios of ${ }^{16} \mathrm{O} /{ }^{17} \mathrm{O}$ ${ }^{12} \mathrm{C} /{ }^{13} \mathrm{C}$ of the grains, of which ${ }^{12} \mathrm{C} /{ }^{13} \mathrm{C}$ ratios are larger than the corresponding solar ratio, are included in the isotopic regions of the mixtures in Fig. 10(c). However, all grains except one have their ${ }^{14} \mathrm{~N} /{ }^{15} \mathrm{~N}-{ }^{12} \mathrm{C} /{ }^{13} \mathrm{C}$ ratios plotted outside the region of the isotopic ratios in Fig. 10(b). As a result, from these diagrams it is still difficult to consider that these grains are expected to be originated from the supernova even if we consider an uncertainty on the chemical compositions in the presupernova stage.

Mixing processes between several distinct layers would occur during the supernova explosion. In such a case, it is expected that there occur new nucleosynthetic processes other than the mechanical mixing between layers and some of the $X$-elements produced in the He-layer are decomposed by protons in the H-rich envelope when the He-layer and the H-rich envelope mix. In the next paper, we will investigate nucleosynthetic processes driven by the mixing between layers in order to find general features of nucleosynthesis in the supernova explosion.

Acknowledgments. The authors would like to thank Kohichi Iwamoto, Ken'ichi Nomoto, and Toshikazu Shigeyama for giving the data of internal structure for a progenitor model, 14E1, and helpful discussion. This work has been supported, in part, by Grandin-Aid for General Scientific Research (B) (Nos. 07454114 and 09440089). The computation has been made by Cray C916 at the Computer Center of Tokyo Institute of Technology.

\section{References}

Amari, S., P. Hoppe, E. Zinner, and R. S. Lewis, Interstellar SiC with unusual isotopic compositions: grains from a supernova?, Astrophys. J., 394, L43-L46, 1992.

Amari, S., E. Zinner, and R. S. Lewis, Large ${ }^{18} \mathrm{O}$ excesses in circumstellar graphite grains from the Murchison meteorite: identification of a massivestar origin, Astrophys. J., 447, L147-L150, 1995.

Amari, S., E. Zinner, and R. S. Lewis, ${ }^{41} \mathrm{Ca}$ in presolar graphite of supernova origin, Astrophys. J., 470, L101-L104, 1996a.

Amari, S., E. Zinner, and R. S. Lewis, A presolar graphite grain with unusual isotopic compositions, Meteoritics \& Planet. Sci. Suppl., 31, A7-A8,
$1996 b$.

Andersen, J., B. Gustafsson, and D. L. Lambert, The lithium isotope ratio in F and G stars, Astron. Astrophys., 136, 65-73, 1984.

Blackmon, J. C., A. E. Champagne, M. A. Hofstee, M. S. Smith, R. G. Downing, and G. P. Lamaze, Measurement of the ${ }^{17} \mathrm{O}(p, \alpha){ }^{14} \mathrm{~N}$ cross section at stellar energies, Phys. Rev. Lett., 74, 2642-2645, 1995.

Boesgaard, A. M. and K. G. Budge, Beryllium abundances in the lithiumdeficient Hyades F stars, Astrophys. J., 338, 875-887, 1989.

Boesgaard, A. M., C. P. Deliyannis, A. Stephens, and D. L. Lambert, Boron in lithium- and beryllium-deficient F stars, Astrophys. J., 492, 727-742, 1998.

Caughlan, G. R. and W. A. Fowler, Thermonuclear reaction rates V, Atom. Data Nucl. Data Tables, 40, 283-334, 1988.

Cayrel, R., M. Spite, F. Spite, E. Vangioni-Flam, M. Cassë, and J. Audouze, New high S/N observations of the ${ }^{6} \mathrm{Li} /{ }^{7} \mathrm{Li}$ blend in HD84937 and two other metal-poor stars, Astron. Astrophys., 343, 923-932, 1999.

Giesen, U., C. P. Browne, J. Görres, J. G. Ross, M. Wiescher, R. E. Azuma, J. D. King, J. B. Vise, and M. Buckby, The influence of low-energy resonances on the reaction rate of ${ }^{18} \mathrm{O}(\alpha, \gamma)^{22} \mathrm{Ne}$, Nuclear Physics, A567, 146-164, 1994.

Hachisu, I., T. Matsuda, K. Nomoto, and T. Shigeyama, Mixing in ejecta of supernovae. II. Mixing width of 2D Rayleigh-Taylor instabilities in the helium star models for type Ib/Ic supernovae, Astron. Astrophys. Suppl., 104, 341-364, 1994.

Hashimoto, M., private communication, 1999.

Hobbs, L. M. and J. A. Thorburn, Lithium isotope ratios in halo stars. II., Astrophys. J., 491, 772-788, 1997.

Hoffman, R. D. and S. E. Woosley, Tables of reaction rates for nucleosynthesis for charged particle, weak, and neutrino interactions $(Z<45)$, http: //isotopes.1bl.gov/isotopes/hw92_1.html, 1992.

Iwamoto, K., T. R. Young, N. Nakasato, T. Shigeyama, K. Nomoto, I. Hachisu, and H. Saio, Instabilities and mixing in SN 1993J, Astrophys. J., 477, 865-875, 1997.

Lamb, S. A., I. Iben, Jr., and W. M. Howard, On the evolution of massive stars through the core carbon-burning phase, Astrophys. J., 207, 209-232, 1976.

Landré, V., N. Prantzos, P. Aguer, G. Bogaert, A. Lefebvre, and J. P. Thibaud, Revised reaction rates for the $\mathrm{H}$-burning of ${ }^{17} \mathrm{O}$ and the oxygen isotopic abundances in red giants, Astron. Astrophys., 240, 85-92, 1990.

Lemke, M., D. L. Lambert, and B. Edvardsson, The boron abundance of Procyon, Pub. Astron. Soc. Pacific, 105, 468-475, 1993.

Lemoine, M., D. N. Schramm, J. W. Truran, and C. J. Copi, On the significance of population II ${ }^{6} \mathrm{Li}$ abundances, Astrophys. J., 478, 554-562, 1997.

Meneguzzi, M., J. Audouze, and H. Reeves, The production of the elements $\mathrm{Li}, \mathrm{Be}, \mathrm{B}$ by galactic cosmic rays in space and its relation with stellar observations, Astron. Astrophys., 15, 337-359, 1971.

Meyer, B. S., T. A. Weaver, and S. E. Woosley, Isotopic source table for a $25 M_{\odot}$ supernova, Meteoritics, 30, 325-334, 1995.

Nittler, L. R., S. Amari, E. Zinner, S. E. Woosley, and R. S. Lewis, Extinct ${ }^{44} \mathrm{Ti}$ in presolar graphite and $\mathrm{SiC}$ : proof of a supernova origin, Astrophys. J., 462, L31-L34, 1996.

Nomoto, K. and M. Hashimoto, Presupernova evolution of massive stars, Phys. Rep., 163, 13-36, 1988.

Nomoto, K., F.-K. Thielemann, and K. Yokoi, Accreting white dwarf models for Type I supernovae. III. Carbon deflagration supernovae, Astrophys. J., 286, 644-658, 1984.

Rebolo, R., L. Crivellari, F. Castelli, B. Foing, and J. E. Beckman, Lithium abundances and ${ }^{7} \mathrm{Li} /{ }^{6} \mathrm{Li}$ ratios in late-type population I field dwarfs, Astron. Astrophys., 166, 195-203, 1986.

Sedov, L. I., Similarity and Dimensional Methods in Mechanics, pp. 260 270, Academic Press, New York and London, 1959.

Shigeyama, T. and K. Nomoto, Theoretical light curve of SN 1987A and mixing of hydrogen and nickel in the ejecta, Astrophys. J., 360, 242-256, 1990

Shigeyama, T., K. Nomoto, M. Hashimoto, and D. Sugimoto, Light-curve models for supernova SN1987A in the Large Magellanic Cloud, Nature, 328, 320-323, 1987.

Smith, V. V., D. L. Lambert, and P. E. Nissen, Isotopic lithium abundances in nine halo stars, Astrophys. J., 506, 405-423, 1998.

Soderblom, D. R., B. F. Jones, S. Balachandran, J. R. Stauffer, D. K. Duncan, S. B, Fedele, and J. D. Hudon, The evolution of the lithium abundances of solar-type stars. III. The pleiades, Astron. J., 106, 1059-1086, 1993.

Thielemann, F.-K., M. Hashimoto, and K. Nomoto, Explosive nucleosynthesis in SN 1987A. II. Composition, radioactivities, and the neutron star 
mass, Astrophys. J., 349, 222-240, 1990.

Thielemann, F.-K., K. Nomoto, and M. Hashimoto, Core-collapse supernovae and their ejecta, Astrophys. J., 460, 408-436, 1996.

Travaglio, C., R. Gallino, S. Amari, E. Zinner, S. E. Woosley, and R. S. Lewis, Low-density graphite grains and mixing in type II supernovae, Astrophys. J., 510, 325-354, 1999.

Weaver, T. A. and S. E. Woosley, Evolution and explosion of massive stars, Ann. New York Acad. Sci., 336, 335-357, 1980.

Weaver, T. A. and S. E. Woosley, Nucleosynthesis in massive stars and the ${ }^{12} \mathrm{C}(\alpha, \gamma){ }^{16} \mathrm{O}$ reaction rate, Phys. Rep., 227, 65-96, 1993.

Woosley, S. E. and T. A. Weaver, The physics of supernova explosions, Annu. Rev. Astron. Astrophys., 24, 205-253, 1986.

Woosley, S. E. and T. A. Weaver, The evolution and explosion of massive stars. II. Explosive hydrodynamics and nucleosynthesis, Astrophys. J. Suppl., 101, 181-235, 1995.

Woosley, S. E., D. H. Hartmann, R. D. Hoffman, and W. C. Haxton, The v-process, Astrophys. J., 356, 272-301, 1990.

Woosley, S. E., R. D. Hoffman, F. X. Timmes, T. A. Weaver, and F.-K. Thielemann, Nucleosynthesis in massive stars and supernovae, Nuclear Physics, A621, 445c-452c, 1997.

Yoshida, T., Light element synthesized in the He-layer and the H-rich envelope of type II supernovae, Doctoral Thesis, 2000.

Yoshida, T., H. Emori, and K. Nakazawa, Light elements synthesized in the He-layer and the H-rich envelope of a Type II supernova - Influence of the adopted neutrino emission model-, Earth Planets Space, 52, 203219, 2000.

Zinner, E., Stellar nucleosynthesis and the isotopic composition of presolar grains from primitive meteorites, Annu. Rev. Earth Planet. Sci., 26, 147$188,1998$.

T. Yoshida (e-mail: tyoshida@gemini.rc.kyushu-u.ac.jp), K. Nakazawa, and H. Emori 